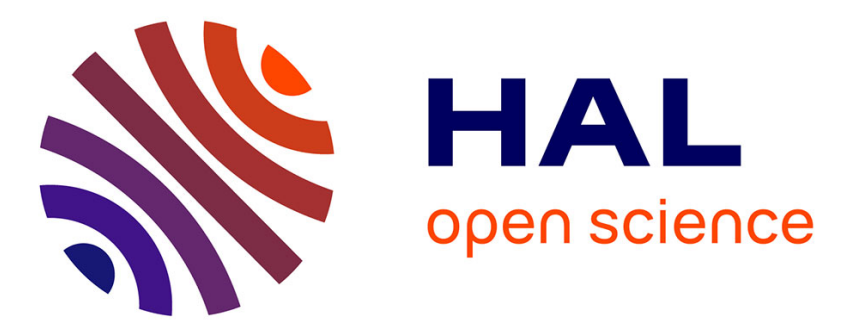

\title{
A hybrid Eulerian Vlasov code. I. Study of high-frequency beatwave experiment and Manley-Rowe action evolution in a finite causal system
}

A. Ghizzo, Pierre Bertrand, J. Lebas, T. W. Johnston, M. Shoucri

\section{- To cite this version:}

A. Ghizzo, Pierre Bertrand, J. Lebas, T. W. Johnston, M. Shoucri. A hybrid Eulerian Vlasov code. I. Study of high-frequency beatwave experiment and Manley-Rowe action evolution in a finite causal system. Physics of Plasmas, 1996, 3 (2), pp.650-668. 10.1063/1.871892 . hal-01791847

\section{HAL Id: hal-01791847 \\ https://hal.univ-lorraine.fr/hal-01791847}

Submitted on 14 May 2018

HAL is a multi-disciplinary open access archive for the deposit and dissemination of scientific research documents, whether they are published or not. The documents may come from teaching and research institutions in France or abroad, or from public or private research centers.
L'archive ouverte pluridisciplinaire HAL, est destinée au dépôt et à la diffusion de documents scientifiques de niveau recherche, publiés ou non, émanant des établissements d'enseignement et de recherche français ou étrangers, des laboratoires publics ou privés. 


\section{A hybrid Eulerian Vlasov code. I. Study of high-frequency beatwave experiment and Manley-Rowe action evolution in a finite causal system}

A. Ghizzo, P. Bertrand, J. Lebas, T. W. Johnston, and M. Shoucri

Citation: Physics of Plasmas 3, 650 (1996); doi: 10.1063/1.871892

View online: https://doi.org/10.1063/1.871892

View Table of Contents: http://aip.scitation.org/toc/php/3/2

Published by the American Institute of Physics

\section{Articles you may be interested in}

A hybrid semi-Lagrangian Vlasov code. II. Numerical simulation of chirped beatwave experiment Physics of Plasmas 5, 4041 (1998); 10.1063/1.873126

Simulation of laser beat heating of a plasma

The Physics of Fluids 18, 470 (1975); 10.1063/1.861153

Two-stage electron acceleration by simultaneous stimulated Raman backward and forward scattering Physics of Plasmas 2, 3115 (1995); 10.1063/1.871144

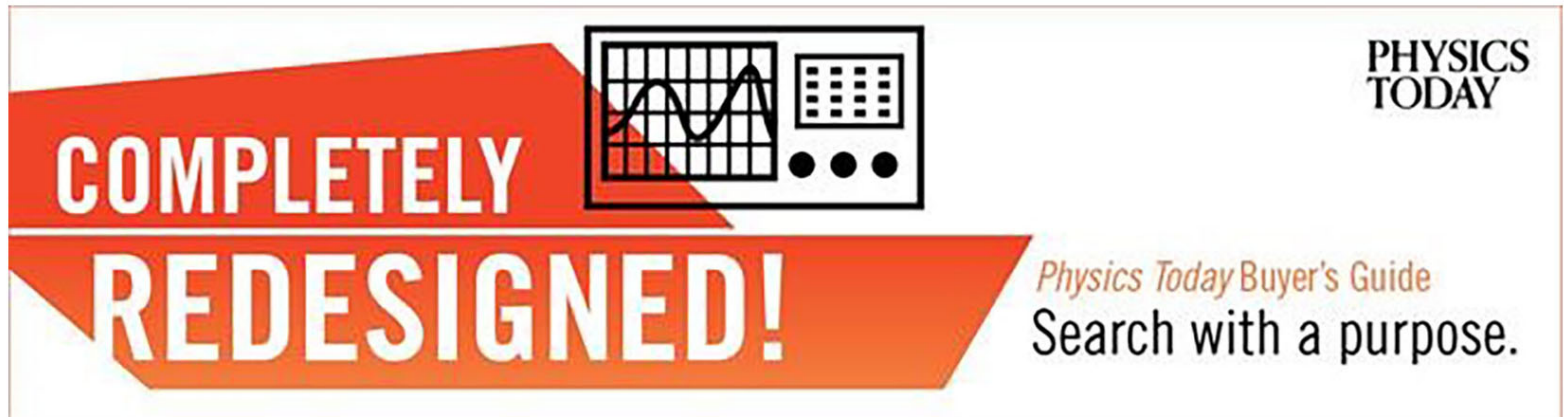




\title{
A hybrid Eulerian Vlasov code. I. Study of high-frequency beatwave experiment and Manley-Rowe action evolution in a finite causal system
}

\author{
A. Ghizzo, P. Bertrand, and J. Lebas \\ Laboratoire de Physique des Milieux Ionisés-C.N.R.S. URA 835-Université de Nancy-I, \\ BP 23954506 Vandoeuvre les Nancy cedex, France \\ T. W. Johnston \\ I.N.R.S. Energie, Université du Québec, Varennes, Canada \\ M. Shoucri \\ Centre Canadien de Fusion Magnétique, Tokamak de Varennes, Varennes, Québec J3X 1S1, Canada
}

(Received 12 July 1995; accepted 1 November 1995)

\begin{abstract}
For the first time, beatwave simulations relevant to the UCLA experiment (University of California at Los Angeles) [see Clayton et al., Phys. Rev. Lett. 70, 37 (1993), and also Phys. Plasmas 1, 1753 (1994)] have been made with a relativistic Eulerian Hilbert-Vlasov code for a realistically high ratio of driver frequency to plasma wave frequency $\left(\omega_{0} / \omega_{p} \approx 30\right)$. Some of the more striking features that have emerged from the Hilbert-Vlasov simulations are discussed in this paper, with particular emphasis on particle dynamics in phase space with beam injection, and action transfer results obtained from the derivation of the integrated Manley-Rowe relations derived for a finite causal system. (C) 1996 American Institute of Physics. [S1070-664X(96)03102-2]
\end{abstract}

\section{INTRODUCTION}

Among the studies that have been undertaken to discover new techniques to accelerate particles up to ultrarelativistic energies, the ones based on the generation of large-amplitude plasma waves (theoretically capable of reaching an electric field of the order of $\mathrm{GeV} / \mathrm{m}$ ) seems very promising. A way to obtain an electric field of such intensities is to inject two electromagnetic waves in a plasma of low density (laser beat wave concept). To obtain fields as strong as possible, the difference of frequencies of the two waves must be chosen to resonate with the plasma frequency, which must be relatively low so that the resulting plasma wave phase velocity will approach light velocity. In these conditions the beat of these two waves induces by resonance a high-phase velocity longitudinal plasma wave, which traps and accelerated electrons to ultrarelativistic energies.

In recent beatwave experiments, the experimental ratios of driver frequency to plasma frequency are very high $\left(\omega_{0} / \omega_{p}=33\right.$ in Ref. 1 and $\omega_{0} / \omega_{p}=100$ in Ref. 2) and this makes severe computer demands for attempts at the simulation of such experiments. These high ratios impose a prohibitive computer burden on a direct attack via Euler-Vlasov ${ }^{3}$ or particle-in-cell (PIC) simulation. (The burden is doubly severe because an exact approach requires both high spatial and time resolution for the very small laser wavelengths and periods, together with the much larger space time scales required for the beatwave effects.) In order to handle such a beatwave experiment, a hybrid solution ${ }^{4}$ has been implemented: the one-and-one-half dimension ( $\left.1 \frac{1}{2}-\mathrm{D}\right)$ Eulerian Vlasov code ${ }^{3}$ has been modified to interface with the highfrequency complex envelopes rather than interfacing directly with the electromagnetic part of the Maxwell equations. Abandoning a detailed description of the high-frequency and high wave vector phenomena, the full Vlasov apparatus is used only for the longitudinal plasma wave aspect of the problem (which gives a precise description of the particle acceleration process), but with the addition of the ponderomotive force driver, which is itself obtained from complex amplitude coupled equations for the electromagnetic driver waves (and any other important electromagnetic sidebands). The time step is thus only a fraction of a plasma period and the spatial resolution is a fraction of light plasma wavelength. It should be noted that backscatter phenomena are thus excluded from the simulation.

For the generation of the complex envelope of plasma wave (which is required at each time step for coupling with the driver waves), we have used the spatial Hilbert transform. In a previous paper ${ }^{4}$ this hybrid model, we have called the Hilbert-Vlasov (HV) code, has been successfully tested in the case of a beatwave experiment with a modest frequency ratio $\omega_{0} / \omega_{p}=2.60$. In such a range of driver frequency, a direct comparison ${ }^{4}$ with the full electromagnetic version of the Vlasov code is then possible. In the simplest case, that of a spatially periodic system with an initial value problem, we have found the two Vlasov codes to agree very well and to exhibit the sames differences of each with respect to the simple nonlinear three-wave envelope model. The action transfer from the plasma wave to accelerated electrons has been evaluated ${ }^{4}$ in the Hilbert-Vlasov code using Manley-Rowe $\mathrm{C}^{5}$ relations and found in good agreement with this obtained in Maxwell-Vlasov simulations.

Some of the more striking features that have emerged from the Hilbert-Vlasov simulations are discussed in this paper, with particular emphasis on particle dynamics and the spatially integrated action transfer (obtained from the Manley-Rowe relations) generalized to the case of a finite causal system. In the submitted companion paper (in Phys. Plasmas) ${ }^{6}$ referred to henceforth as II, the beatwave-driven plasma wave limiting by relativistic detuning is investigated, including beat frequency chirping, i.e., evade the detuning.

We present here some examples of the use of the HV code at high-frequency ratios, together with a comparison (as 
in Ref. 4) with the full electromagnetic version of the code-the Maxwell-Vlasov-code (at modest frequency ratios where both codes can be run). The presentation of the work is as follows: in Sec. II the HV code is discussed and in Sec. III the Manley-Rowe relations for the action conservation along the various wave characteristics are derived in order to obtain an accurate estimate of action transferred to particles. In Sec. IV, numerical simulations have been carried out with an incident beatwave driver at a modest value for the frequency ratio (pump wave frequency close to $\omega_{0}=2.60 \omega_{p}$ ), allowing close comparison between the two kinds of Vlasov codes (the comparison with a simple nonlinear three-wave envelope code including linear loss and approximative relativistic detuning is presented in Ref. 4). Section $\mathrm{V}$ contains an example of high-frequency beatwave $\left(\omega_{0} \approx 30 \omega_{p}\right)$ using the HV code. Also included is the straightforward extension of the previous analysis to the case of an electron beam injection into the plasma, which leads to a strong particle acceleration process. Finally, we examine in Sec. VI an extended simulation, including the downcascading process (where the Stokes wave decays as a pump) for a value of the driver frequency of $\omega_{0}=3.66 \omega_{p}$ (to limit the process to one step), with close comparison with the Maxwell-Vlasov simulations. Section VII contains some discussions and conclusion.

\section{THE RELATIVISTIC HILBERT-VLASOV MODEL}

In this section we discuss the implementation of a hybrid solution (the HV code) to handle one-dimensional beatwave experiments with very high-frequency driver waves. The 1 $\frac{1}{2}$-D Eulerian Maxwell-Vlasov code is modified to interface with the high-frequency complex envelopes rather than interfacing directly with the electromagnetic part of the Maxwell equations. The ponderomotive force for the Vlasov code is then computed from the complex amplitude equations. The complex density envelope required to calculate the complex beatwave source current for each high-frequency wave is obtained from the real Vlasov output, which is converted to a complex envelope from the use of the fast spatial Hilbert transform. We present here the two kinds of Vlasov codes.

\section{A. The full electromagnetic Maxwell-Vlasov code}

We now derive the equations that govern the full electromagnetic version of the Vlasov code. We assume that the Vlasov equation is relativistic in the longitudinal (and acceleration) direction $x$. For the linear ( $y$ direction), laser polarization intensities we consider, the transverse dynamics can be economically included in the nonrelativistic transverse cold fluid approximation. The Vlasov equation in the acceleration direction is thus

$$
\frac{\partial f}{\partial t}+\frac{p_{x}}{m \gamma} \frac{\partial f}{\partial x}-e\left[E_{x}+u_{y}(x, t) B_{z}(x, t)\right] \frac{\partial f}{\partial p_{x}}=0,
$$

where $\gamma=\left(1+p_{x}^{2} / m^{2} c^{2}\right)^{1 / 2}$ is the Lorentz factor. In the $y$ direction, for reasons of computer economy, the fluid transverse momentum nonrelativistic $P_{y}=m u_{y}$ satisfies the following equation:

$$
\frac{\partial P_{y}}{\partial t}=-e E_{y} .
$$

These two equations, together with the usual onedimensional $(x)$ Maxwell equations, makes up the system of equations used the model we called the Maxwell-Vlasov code. The details of the way in which the equations are advanced and the boundary conditions are imposed have been extensively described elsewhere. ${ }^{7,8}$

\section{B. The Hilbert-Vlasov code}

As we have mentioned earlier, we replace the Maxwell's equations for transverse waves by a three-wave model coupled to the Vlasov equation, in order to handle the highfrequency electromagnetic waves (for which full detail would require calculations over very short intervals in time and space). This has been greatly facilitated by the noiseless character of the Vlasov code, which allows an accurate calculation of the complex amplitude using the Hilbert transform. In a resonant coupling mechanism of the kind encountered in classical three-wave interaction processes (such as beatwave process or stimulating Raman scattering), the vector potential $A_{y}$ of the electromagnetic wave is in the usual form:

$$
A_{y}(x, t)=\frac{1}{2} A_{0}(x, t) e^{i\left(k_{0} x-\omega_{0} t\right)}+\frac{1}{2} A_{s}(x, t) e^{i\left(k_{s} x-\omega_{s} t\right)}+\text { c.c. },
$$

where " 0 " and " $s$ " refer, respectively, to the pump and idler (Stokes) components of the wave vector (in a beatwave process the anti-Stokes component is negligible, however, the method can be extended to more complex situations including more sidebands in the envelope equations, as, for instance, in cascade processes).

The envelope equations for $A_{0}$ and $A_{s}$ can then be written with $\omega_{0}=\omega_{e}+\omega_{s}$ and $k_{0}=k_{e}+k_{s}$, in which " $e$ " refers to the electron plasma wave:

$$
\begin{aligned}
& \left(\frac{\partial}{\partial t}+\nu_{g 0} \frac{\partial}{\partial x}\right) A_{0}=-\frac{i \omega_{p}^{2}}{4 \omega_{0} n_{0}} A_{s} \rho_{e}, \\
& \left(\frac{\partial}{\partial t}+\nu_{g s} \frac{\partial}{\partial x}\right) A_{s}=\frac{i \omega_{p}^{2}}{4 \omega_{s} n_{0}} A_{0} \rho_{e}^{*},
\end{aligned}
$$

where $n_{0}$ and $\rho=\frac{1}{2} \rho_{e}(x, t) e^{i\left(k_{e} x-\omega_{e} t\right)}+$ c.c. denote the homogeneous electron density and the perturbed density, respectively. The group velocities are the standard values for electromagnetic waves in plasma: (with $i=0, s$ ), we have

$$
\frac{\nu_{g i}}{c}=\left(1-\frac{\omega_{p}^{2}}{\omega_{i}^{2}}\right)^{1 / 2} .
$$

In the Hilbert-Vlasov model we integrate the modified Vlasov equation, including the ponderomotive force component:

$$
\frac{\partial f}{\partial t}+\frac{p_{x}}{m \gamma} \frac{\partial f}{\partial x}+\left[-e E_{x}+F(x, t)\right] \frac{\partial f}{\partial p_{x}}=0 .
$$

Both Vlasov and envelope equations are integrated using a time-splitting scheme of the usual kind (see Ref. 4). The 


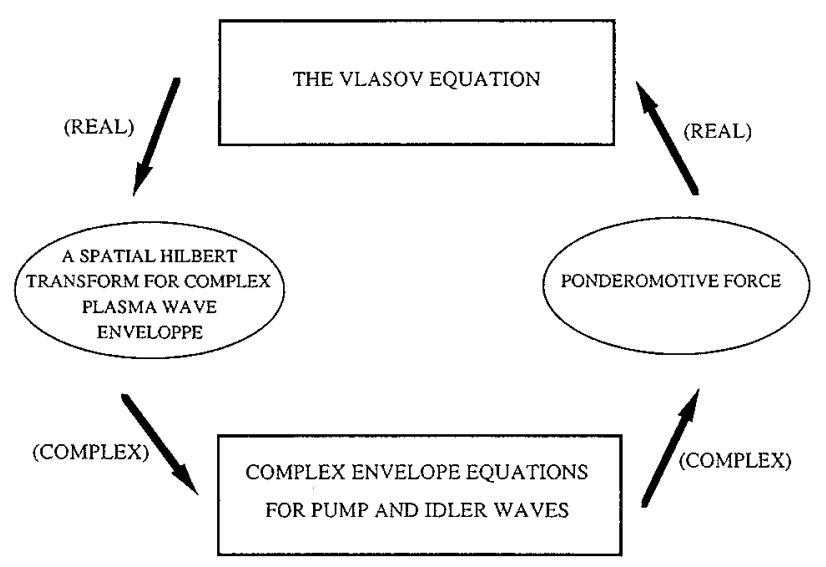

FIG. 1. Global scheme of the Hilbert-Vlasov code. Here the Maxwell equations for transverse waves are replaced by the three-wave model [Eq. (4)] coupled to the Vlasov equation used for the description of the electron plasma wave. The beat force for the Vlasov equation is computed from the complex amplitudes of driver components via the ponderomotive force [Eq. (7)]. On the other hand, the longitudinal plasma field is converted to a complex envelope amplitude required for coupled mode equations via a spatial Hilbert transform.

different step used for integrating the Vlasov and envelope equations are graphically illustrated in Fig. 1.

The interface between the envelope equations (which require the knowledge of the complex amplitude for the rapidly oscillating phases) and the Vlasov equation can be realized by using a spatial Hilbert transform to compute the complex density envelope $\rho_{e}$. Second, the Lorentz force is replaced by the real ponderomotive force to calculate the electron distribution function via the Vlasov equation, using the following relation:

$$
F(x, t)=\frac{e^{2}}{2 m} \frac{\partial\left(A_{y}^{2}\right)}{\partial x}=\frac{e^{2}}{4 m}\left(i k_{e} A_{0} A_{s}^{*} e^{i\left(k_{e} x-\omega_{e} t\right)}+\text { c.c. }\right) .
$$

Extension of the previous analysis to the case where we have to take into account the anti-Stokes mode (as in a forward Raman scattering) or to look into cascading, is then straightforward. This model provides a saving of order $\left(\omega_{0} / \omega_{p}\right)^{2}$ (i.e., time $x$ space) in computer time, as compared with the direct Maxwell-Vlasov with the highest space-time resolution required.

\section{RAMAN SCATTERING AND MANLEY-ROWE RELATIONS IN A FINITE CAUSAL SYSTEM}

We will now analyze Raman scattering and the action transfer to accelerated particles in some details. In a previous work, ${ }^{4}$ forward Raman scattering has been investigated by using Maxwell-Vlasov and Hilbert-Vlasov simulations using periodic boundary conditions. The main interest of these periodic boundary conditions is to allow a direct comparison with theoretical predictions as the conservation of density action sums, well known as the Manley-Rowe invariants. Action conservation was satisfactorily verified for the two significant electromagnetic waves (pump and scattered waves) for Maxwell-Vlasov simulations (while in the case of the Hilbert-Vlasov model, they are exactly conserved since computed via the three-wave model). We were also able to account very well for the plasma wave action loss by using the very detailed phase space diagnostics afforded by the Eulerian Vlasov codes, together with plasma wave separatrices to allow classification of particle energy and action transfer from the plasma wave to electrons. The action loss between the pump and plasma wave was shown to be well accounted for in detail by examining the evolution energy for electrons above the lower separatrix in phase space. We found the two Vlasov codes to agree very well in giving much the same estimate of action transferred to electrons.

In a more realistic long open system with external sources, which we characterize as "causal," we began to see some modest but noticeable differences between the two kinds of Vlasov codes, which are, however, still much closer to each other than to the coupled wave model. In order to make this point precisely and therefore to compare in detail both Vlasov codes, Manley-Rowe relations for the action density conservation along the various wave characteristics are derived in a finite causal system. To compare exactly the results obtained by the HV code with those given by the full electromagnetic version, it is then necessary to use a fourwave coupled mode system to take into account the antiStokes growth, even though this mode remains at a relatively weak level in the beatwave experiment. (Note that in the beatwave process the anti-Stokes mode is attenuated rather than amplified. We shall see later that the action density of the anti-Stokes mode is close to $10 \%$ of the maximum level of the pump action density.) This accurate estimate of action transferred to high accelerated electrons allows us to check the validity of our HV model. We wish to analyze if the method of coupling the longitudinal Vlasov code with the ponderomotive force generated by the beating between the transverse waves (which are treated by usual slowly varying envelope approximation) will modify the plasma wave level and hence the particle acceleration.

Defining the complex action amplitude $a_{(0, s, \mathrm{aS}, e)}$ such that the action density $S=a a^{*}$ is given from the energy density $W$ by

$$
S=\frac{W}{\omega}=\frac{\langle\text { energy density }\rangle_{\omega}}{\omega},
$$

the four-wave envelope equations can be written in the following form:

$$
\begin{aligned}
& \left(\frac{\partial}{\partial t}+\nu_{g 0} \frac{\partial}{\partial x}\right) a_{0}=-\Gamma_{s} a_{s} a_{e}+\Gamma_{\mathrm{aS}} a_{\mathrm{aS}} a_{e}^{*} e^{i \delta_{\mathrm{aS}} x} \\
& \left(\frac{\partial}{\partial t}+\nu_{g s} \frac{\partial}{\partial x}\right) a_{s}=\Gamma_{s} a_{0} a_{e}^{*} \\
& \left(\frac{\partial}{\partial t}+\nu_{g \mathrm{aS}} \frac{\partial}{\partial x}\right) a_{\mathrm{aS}}=-\Gamma_{\mathrm{aS}} a_{0} a_{e} a^{-i \delta_{\mathrm{aS} x} x} \\
& \left(\frac{\partial}{\partial t}+\nu_{g e} \frac{\partial}{\partial x}+\gamma+i \Delta_{\mathrm{rel}}\left|a_{e}\right|^{2}\right) a_{e}=\Gamma_{s} a_{0} a_{s}^{*}+\Gamma_{\mathrm{aS}} a_{\mathrm{aS}} a_{0}^{*} e^{i \delta_{\mathrm{as}} x}
\end{aligned}
$$

where $a_{i}=\left(\epsilon_{0} \omega_{i} / 2\right)^{1 / 2} A_{i} \quad$ for $\quad i=0, \quad s, \quad$ aS $\quad$ and $a_{e}=\left(\epsilon_{0} \omega_{e} / 2\right)^{1 / 2}\left(i \rho_{e} / k_{e} \omega_{p}\right)$. Here the relativistic correction 
retained in the plasma envelope equation is $\Delta_{\text {rel }}=\left(3 / 4 \omega_{p}^{2} \epsilon_{0}\right)\left(e \omega_{e} / m c\right)$ and the coupling coefficient are $\Gamma_{s ; \mathrm{aS}}=(e / 2 m)\left(2 \epsilon_{0} \omega_{0} \omega_{s ; \mathrm{aS}} \omega_{e}\right)^{-1 / 2} k_{e} \omega_{p}$. The subscripts " 0 ," "s," "aS," or " $e$ " are, respectively, related with the pump, idler, anti-Stokes, and electron plasma wave, while $\delta_{\mathrm{aS}}=k_{0}+k_{e}-k_{\mathrm{aS}}$ denotes the wave vector mismatch for the anti-Stokes mode (no mismatch is introduced for the idler wave). Multiplying Eqs. (8)-(11), respectively, by $a_{0}^{*}, a_{s}^{*}$, $a_{\mathrm{aS}}^{*}$, and $a_{e}^{*}$, the corresponding complex conjugate equation by $a_{0}, a_{s}, a_{\mathrm{aS}}$, and $a_{e}$ and by appropriate summing, we obtain the following three relations (only two of which are independent), which are a four-wave version of the usual Manley-Rowe relations:

$$
\begin{aligned}
& \left(\frac{\partial}{\partial t}+\nu_{g 0} \frac{\partial}{\partial x}\right) a_{0} a_{0}^{*}+\left(\frac{\partial}{\partial t}+\nu_{g s} \frac{\partial}{\partial x}\right) a_{s} a_{s}^{*} \\
& +\left(\frac{\partial}{\partial t}+\nu_{g \mathrm{aS}} \frac{\partial}{\partial x}\right) a_{\mathrm{aS}} a_{\mathrm{aS}}^{*}=0, \\
& \left(\frac{\partial}{\partial t}+\nu_{g e} \frac{\partial}{\partial x}+2 \gamma\right) a_{e} a_{e}^{*}-\left(\frac{\partial}{\partial t}+\nu_{g s} \frac{\partial}{\partial x}\right) a_{s} a_{s}^{*} \\
& +\left(\frac{\partial}{\partial t}+\nu_{g \mathrm{aS}} \frac{\partial}{\partial x}\right) a_{\mathrm{aS}} a_{\mathrm{aS}}^{*}=0, \\
& \left(\frac{\partial}{\partial t}+\nu_{g 0} \frac{\partial}{\partial x}\right) a_{0} a_{0}^{*}+\left(\frac{\partial}{\partial t}+\nu_{g e} \frac{\partial}{\partial x}+2 \gamma\right) a_{e} a_{e}^{*} \\
& +2\left(\frac{\partial}{\partial t}+\nu_{g \mathrm{aS}} \frac{\partial}{\partial x}\right) a_{\mathrm{aS}} a_{\mathrm{aS}}^{*}=0 .
\end{aligned}
$$

Note that these relations are independent of $\delta_{\mathrm{aS}}$ and $\Delta_{\mathrm{rel}}$. Integrating Eq. (12) over the space between $x=0$ and $x=L$ yields

$$
\begin{aligned}
& \frac{\partial}{\partial t}\left(N_{0}+N_{s}+N_{\mathrm{aS}}\right)+\frac{\nu_{g 0}}{\hbar} \int_{0}^{L} \frac{\partial}{\partial x}\left(a_{0} a_{0}^{*}\right) d x \\
& +\frac{\nu_{g s}}{\hbar} \int_{0}^{L} \frac{\partial}{\partial x}\left(a_{s} a_{s}^{*}\right) d x+\frac{\nu_{g \mathrm{aS}}}{\hbar} \int_{0}^{L} \frac{\partial}{\partial x}\left(a_{\mathrm{aS}} a_{\mathrm{aS}}^{*}\right) d x=0
\end{aligned}
$$

where

$$
\int_{0}^{L} a_{i} a_{i}^{*} d x=\hbar N_{i}, \quad \text { with } i={ }^{\prime}(0, s, \mathrm{aS}, e,,
$$

$N_{0}, N_{s}$, and $N_{\mathrm{aS}}$ represent the mean number of pump, idler, and anti-Stokes photons in the slab per unit area, respectively, while $N_{e}$ represents the mean number of plasmons per unit area. The consequence of these spatially integrated Manley-Rowe relations may be then best appreciated by rewriting Eq. (15) in the following form:

$$
\frac{d}{d t}\left(N_{0}+N_{s}+N_{\mathrm{aS}}\right)=F_{0}+F_{s},
$$

where the quantity $F_{i}=\left(\nu_{g i} / \hbar\right)\left[S_{i}(x=0, t)-S_{i}(x=L, t)\right]$ $=\left(\nu_{g i} / \hbar\right)\left[a_{i} a_{i}^{*}(x=0, t)-a_{i} a_{i}^{*}(x=L, t)\right]$ denotes the photon flux of type $i$ entering the slab at $x=0$, less that exiting at
$x=L$. This exit flux is zero here because the simulation stops before the right border is reached. In a similar way, Eqs. (13) and (14) lead to the respective relations:

$$
\frac{d}{d t}\left(N_{e}-N_{s}+N_{\mathrm{aS}}\right)+2 \gamma N_{e}=-F_{s}
$$

and

$$
\frac{d}{d t}\left(N_{0}+N_{e}+2 N_{\mathrm{aS}}\right)+2 \gamma N_{e}=F_{0} .
$$

Rewriting Eq. (9), we obtain the form useful for checking action transfer to electrons:

$$
2 \gamma N_{e}=F_{0}-\frac{d}{d t}\left(N_{0}+N_{s}+2 N_{\mathrm{aS}}\right) .
$$

In the lossless Vlasov plasma, with a single plasma wave being responsible for the energy transferred to fast electrons, we hypothesized that the kinetic (relativistic) energy of all the electrons above the lower momentum boundary of the wave instantaneous separatrix (see Ref. 5 for more detail) would account for the energy missing and (on division by the electron plasma wave frequency and $\hbar$ ) for the corresponding missing "mean number of plasmons of wave-particle interaction (per unit area)." Thus, defining

$$
N_{\mathrm{WP}}=\frac{m c^{2}}{\hbar \omega_{e}} \int_{0}^{L} d x \int_{p_{\text {low }}}^{+\infty} d p_{x}(\gamma-1) f\left(x, p_{x}, t\right),
$$

Eqs. (18) and (19) can be rewritten in the following form:

$$
\begin{aligned}
& \frac{d}{d t}\left(N_{e}+N_{\mathrm{WP}}-N_{s}+N_{\mathrm{aS}}\right)=-F_{s}, \\
& \frac{d}{d t}\left(N_{0}+N_{e}+N_{\mathrm{WP}}+2 N_{\mathrm{aS}}\right)=F_{0} .
\end{aligned}
$$

\section{COMPARISON OF THE TWO KINDS OF VLASOV SIMULATIONS}

The Hilbert-Vlasov model described above is now applied to a beatwave experiment in a finite causal system, with an externally incident beatwave driver at a modest value for the pump wave frequency, allowing close comparison between the HV model and the Maxwell-Vlasov model.

The incident electromagnetic waves $\left(\omega_{0}, k_{0}\right)$ (for the pump wave) and $\left(\omega_{s}, k_{s}\right)$ (for the idler wave), continuously generated at $x=0$, beat together inside the plasma to produce a forward-going electron longitudinal plasma wave $\left(\omega_{e}, k_{e}\right)$ according to the matching conditions $\omega_{0}=\omega_{s}+\omega_{e}$ and $k_{0}=k_{s}+k_{e}$ for frequency and wave numbers, respectively. The wave propagation parameters used for the comparison are summarized in Table I. To compare directly with our previous work, ${ }^{4}$ the plasma was chosen with two electron temperature components, the majority (95\%) component with a $15 \mathrm{keV}$ temperature and a minority component $(5 \%)$ at $100 \mathrm{keV}$. The cold component electron temperature is high enough for electron Landau damping to subdue the usually rapidly growing (but here unwanted) backward Raman scattering instability, while the hot component temperature is high enough to ensure sufficient electrons to study electron 
TABLE I. Numerical parameters used in the comparison between the Hilbert-Vlasov and the Maxwell-Vlasov codes.

\begin{tabular}{lccc}
\hline \hline & Pump wave $(0)$ & Idler wave $(s)$ & Plasma wave $(e)$ \\
\hline Frequency $\omega / \omega_{p}$ & 2.60 & 1.534 & 1.065 \\
Wave number $k c / \omega_{p}$ & 2.40 & 1.164 & 1.236 \\
Group velocity $\nu_{g} / c$ & 0.923 & 0.759 & 0.102 \\
Quiver $\nu_{\text {osc }(o, s)} / c$ & 0.0870 & 0.1475 & 0.861 \\
Phase $\nu_{\varphi} / c$ velocity & & & \\
Quiver $p_{\text {osc }(o, s)} / m c$ & 0.0873 & 0.149 & 1.697 \\
Phase $p_{\varphi} / m c$ & & & \\
Momentum & & & \\
\hline \hline
\end{tabular}

acceleration by the forward Raman scattering, which is relevant to beatwave acceleration. The two Vlasov codes ${ }^{4}$ have been found to agree very well and to exhibit the same differences of each other with respect to the standard coupledmode model, which is unable to describe correctly the damping of the plasma wave due to its phenomenological description by a constant linear damping. Here the emphasis is put on the study of nonlinear behavior of large systems and over long times, and also on Manley-Rowe action considerations. The numerical simulations were performed with two laser beams, with normalized frequency $\omega_{0}=2.60 \omega_{p}$ and $\omega_{s}=1.534 \omega_{p}$ injected into the plasma at the left of the system $(x=0) \quad$ with equal amplitude $E_{0} / \sqrt{2}$ with $e E_{0} / m \omega_{p} c=0.32$. Wave envelopes rise to these values through a rise time profile of the type $\sin ^{2}\left(\pi t \omega_{p} / 100\right)$. Since energy densities are normalized to $n_{0} m c^{2}$, the frequency to $\omega_{p}$, the action density $S_{0}$ is then normalized by division with $n_{0} m c^{2} / \omega_{p}$ so that the normalized pump action density is

$$
\frac{S_{0} \omega_{p}}{n_{0} m c^{2}}=\frac{\omega_{p}}{2 \omega_{0}}\left(\frac{e E_{0}}{m c \omega_{p} \sqrt{2}}\right)^{2}
$$

i.e. an initial value of 0.00984 . The idler action density is equal to $S_{s} \omega_{p} / n_{0} m c^{2}=0.0167$. The flux of photons injected into the plasma is then

$\frac{F_{0}}{n_{0} c} \frac{\hbar \omega_{p}}{m c^{2}}=\frac{\nu_{g 0}}{c} \frac{S_{0} \omega_{p}}{n_{0} m c^{2}}=0.00908$, for the pump wave,

$\frac{F_{s}}{n_{0} c} \frac{\hbar \omega_{p}}{m c^{2}}=\frac{\nu_{g s}}{c} \frac{S_{s} \omega_{p}}{n_{0} m c^{2}}=0.0126$ for the idler wave.

The spatial envelopes of the different modes of the rapidly oscillating fields were obtained using a Hilbert transform $^{4-9}$ (specifically, at a given time, the signal is Fourier analyzed and then a spatial Hilbert transform is performed to obtain the quadrature component leading to the envelope of the fields). Figure 2(a) shows the Hilbert envelopes of the three different electromagnetic modes together with the plasma component in density action units, at time $t \omega_{p}=900$, computed in the Maxwell-Vlasov code, while Fig. 2(b) shows the direct envelopes computed by the HV code.

It is clear that the general features of the different modes are well reproduced by the Hilbert-Vlasov model, particularly for the electromagnetic waves. The interaction of the electromagnetic drivers (pump and idler waves) is strong enough for the four modes to be present. (Note that the anti-

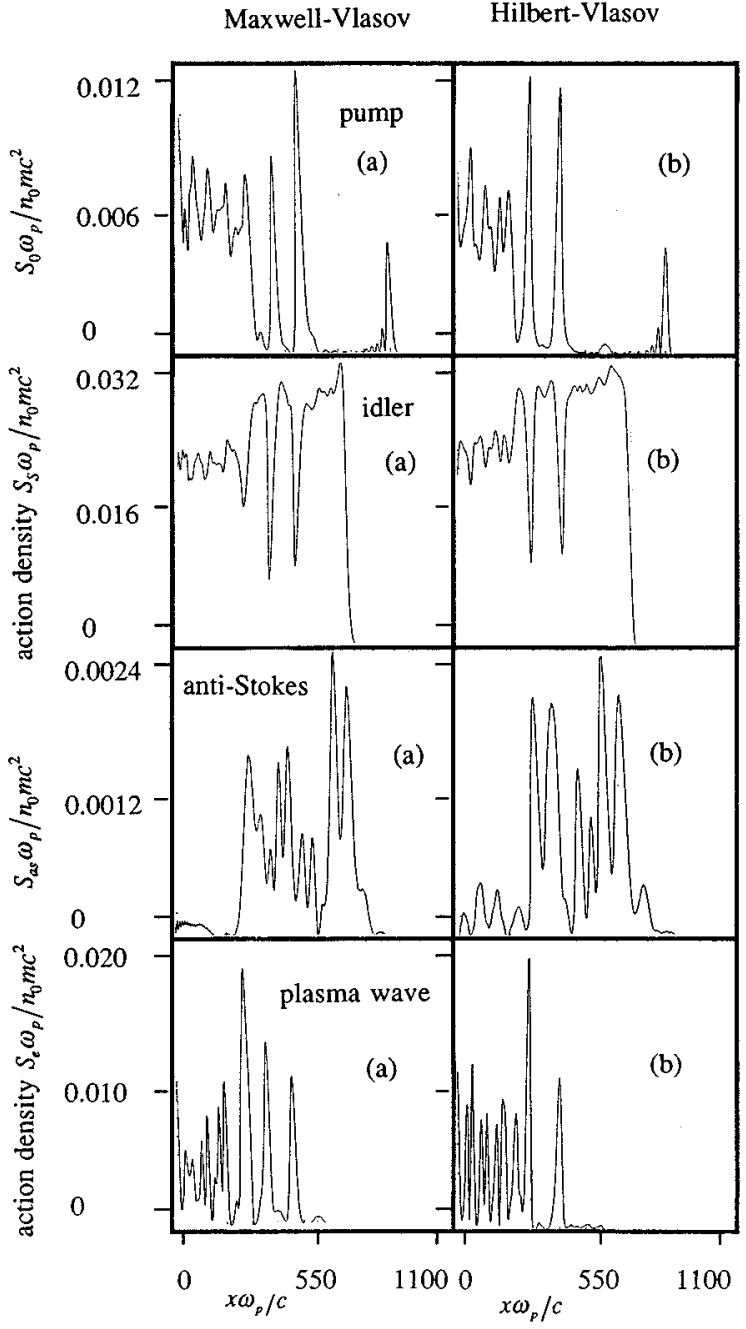

FIG. 2. Display at time $t \omega_{p}=900$ of the different modes (pump, idler, antiStokes, and plasma wave) in normalized density action units: (a) obtained using a spatial Hilbert transform of the electromagnetic field in the Maxwell-Vlasov (full electromagnetic) code; (b) obtained in the hybrid version of the code, i.e. the Hilbert-Vlasov code.

Stokes mode remains at a weak level in comparison with the driver modes.) There is a series of violent pump depletions and idler increases, together with strong amplitude modulations of the plasma wave. Note the occurrence of intense peaks in pump behavior reaching a level higher than the value initially injected into the plasma, corresponding to a strong decrease of the idler wave (which now plays the role of an "antipump" in a system capable of transferring energy back to the pump wave).

The maximum amplitudes of the electromagnetic waves are roughly the same in the two kinds of Vlasov simulations. There are however, some distinct differences in shape and in the positions of the peaks of the pump wave, indicating dephasing in time. Nonetheless, these results show clearly that the coupling of four-wave model with the Vlasov equation constituting the hybrid model seems to account rather well for the nonlinear and complex behavior observed in the full electromagnetic version of the Vlasov code in this rather violent interaction. Nonetheless, there are significant differences in the plasma wave. 

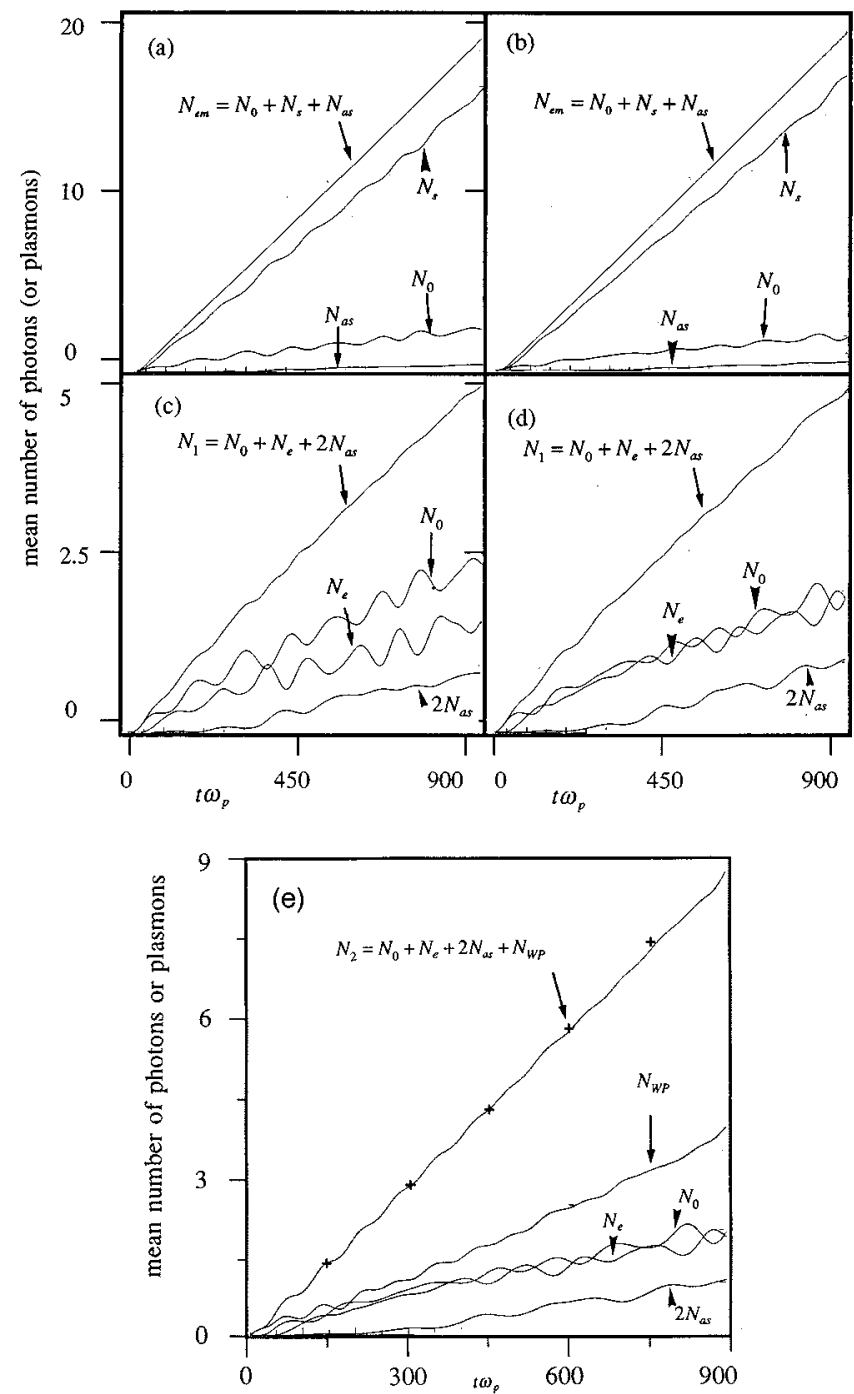

FIG. 3. Temporal behavior of the normalized mean number of photons (per area unit) $N_{0}, N_{s}, N_{\mathrm{aS}}$ for the pump, idler, and anti-Stokes components, respectively, and the mean number of plasmons $N_{e}$ created by the plasma wave and their mutual sums $N_{\mathrm{em}}=N_{0}+N_{s}+N_{\mathrm{aS}}$ for the full electromagnetic version of the model in (a) and of the Vlasov-Hilbert code in (b) and of the quantity $N_{1}=N_{0}+N_{e}+2 N_{\mathrm{aS}}$ for the full electromagnetic version in (c) and the corresponding result given by the HV model in (d). (e) The same as in (d), but now including the mean number of equivalent plasmons $N_{\text {WP }}$ inferred from electron acceleration by wave-particle interactions in the components in the case of Hilbert-Vlasov simulation. One see clearly that adding $N_{\mathrm{WP}}$ to the sum $N_{1}=N_{0}+N_{e}+2 N_{\mathrm{aS}}$ [shown in (d)] to compute the quantity $N_{2}=N_{1}+N_{\text {WP }}$ now yields the correct linear behavior with time, as expected.

To understand the behavior of trapped particle dynamics and to estimate the efficiency of the action transfer, diagnostics employing Manley-Rowe action relations are performed. Looking at the temporal action density evolution gives a more precise comparison between both methods.

Let us begin with electromagnetic action density. The time evolution of the mean number of photons (per area unit) $N_{0}$ (for pump), $N_{s}$ (for idler), $N_{\mathrm{aS}}$ (for anti-Stokes wave) obtained from the Maxwell-Vlasov simulation, together with their sum $N_{\mathrm{em}}=N_{0}+N_{s}+N_{\mathrm{aS}}$ is shown in Fig. 3(a) (which is equivalent to the first electromagnetic Manley-
Rowe invariant for the periodic system). As expected, $N_{\mathrm{em}}$ behaves linearly with time with a numerical slope evaluated to be $F_{\text {num }} \hbar \omega_{p} / n_{0} m c^{3}=0.0218$, i.e. very close to the total photon flux injected in plasma $\left(F_{0}+F_{s}\right) \hbar \omega_{p} / n_{0} m c^{3}$ $=0.0217$. Here the Hilbert transform has been used as a simple diagnostic to aid comparison with theory. A very useful bridge between simulation and theory is thus provided by the time evolution of the complex space envelopes calculated from spatial Hilbert transforms. It was the success of these comparisons together with the noiseless character of the Eulerian Vlasov scheme that led us to the HV method. The corresponding figure obtained by the HV model is shown in Fig. 3(b), indicating a very close correspondence for the electromagnetic invariant.

In Fig. 3(c) and Fig. 3(d) we present the evolution with time of $N_{0}$, of $N_{\mathrm{aS}}$ (multiplied by a factor 2) and of the mean number of plasmons (per area unit) $N_{e}$ obtained by the Maxwell-Vlasov code and the Hilbert-Vlasov code, respectively, and here the differences in the two codes are quite noticeable. Because of the dynamics of the particle acceleration, "the mean number of photon plus plasmon sum" has a more complicated behavior, which is nonlinear with time. Significant temporal variations take place for the quantities $N_{e}$ and $N_{0}$, but the total quantity $N_{1}=N_{0}+N_{e}+2 N_{\text {aS }}$ presents a similar evolution in both codes. Thus, a deviation from the linear behavior appears simultaneously, due to details of electron trapping. While there is a slight difference in $N_{1}$, the chief difference in the two simulations is in the relative amounts of $N_{0}$ and $N_{e}$. The HV code gives nearly equal levels rather than the Maxwell-Vlasov ratio of about 1.5, with rather high oscillations. Considering the violence of the interactions, the codes compare very well. To check this point in the lossless Hilbert-Vlasov code, we compute the missing action by considering the relativistic kinetic energy of all accelerated particles above the lower separatrix momentum (and on division by the electron plasma frequency and $\hbar$ ) to obtain finally the mean number of plasmons of wave-particle interaction $N_{\text {WP }}$ according to Eq. (23). In Fig. 3(e), where $N_{\text {WP }}$ is given with the components of Fig. 3(d), one can see clearly that adding $N_{\mathrm{WP}}$ to the sum $N_{1}=N_{0}+N_{e}+2 N_{\mathrm{aS}}$ of Fig. 3(d) indeed gives small temporal oscillation around the straight line, whose slope given by 0.0097 is very close to the theoretical value $F_{0}\left(\hbar \omega_{p} / n_{0} m c^{3}\right)=0.0091$. We have plotted some values of the theoretical quantity $N_{2}=N_{1}+N_{\text {WP }}$. (Although the previous analysis has been performed in the case of the HilbertVlasov model, this one can also be investigated for the full electromagnetic version of the Vlasov model.) Thus, the nonlinear absorption $F_{0} \hbar \omega_{p} / n_{0} m c^{3}=0.0091$ due to particle trapping can be separated from the coupled mode effects, and leads to an estimation of energy transfer. The action transferred to accelerated particles can be thus easily measured at each time step using again a spatial Hilbert transform of the electric potential to compute the separatrix boundaries. In conclusion, as remarked above, the good agreement of the "electromagnetic action density" (of linear variation of $N_{\mathrm{em}}=N_{0}+N_{s}+N_{\mathrm{aS}}$ with time) allows us to handle highfrequency problems with complex amplitude equations for the pump, idler, and the anti-Stokes waves, with the Vlasov 

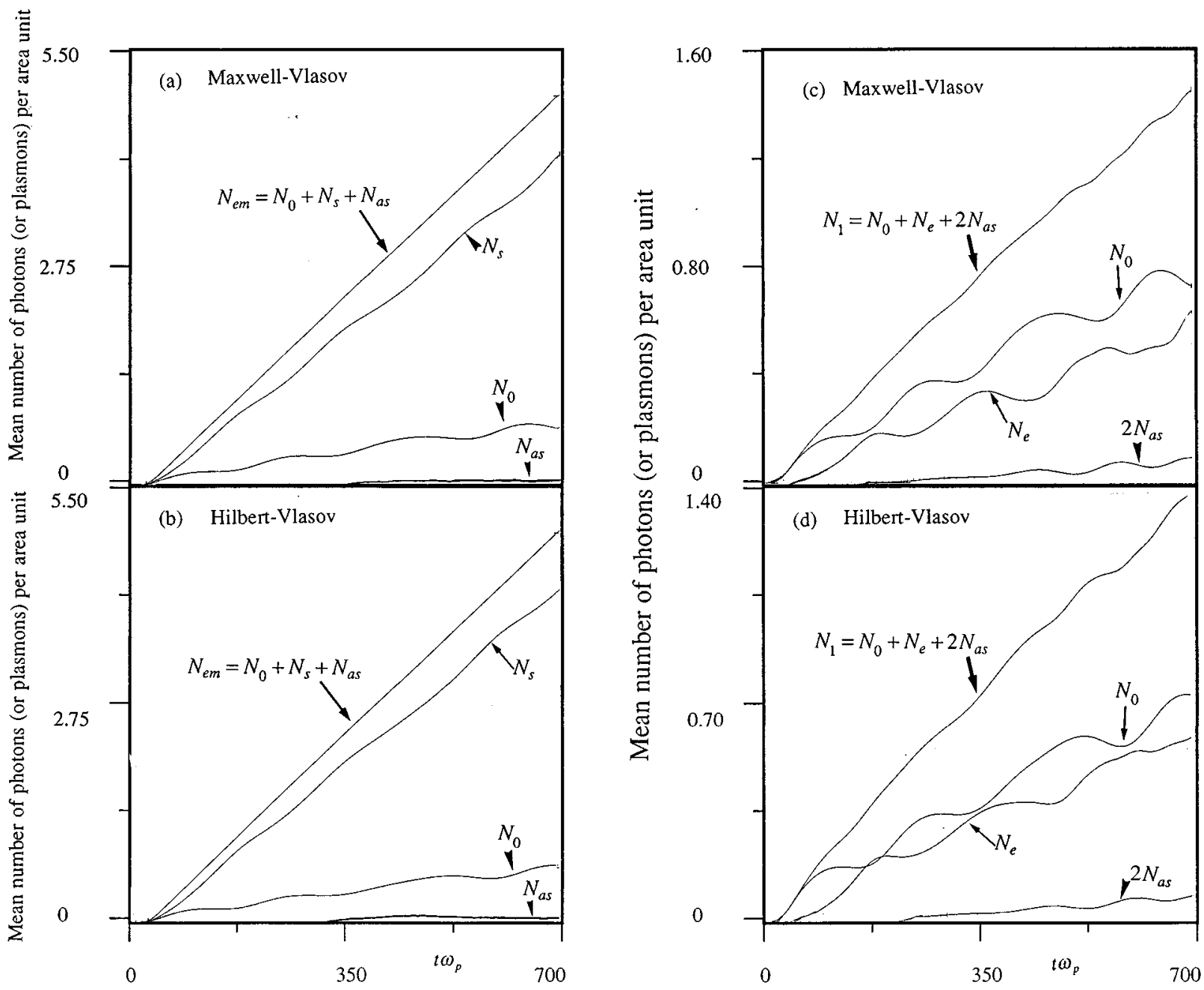

FIG. 4. A case similar to Fig. 3, except the electromagnetic field amplitude is weaker $\left(e E_{0} / m \omega_{p} c=0.188\right)$, showing close agreement between both codes. Decreasing the amplitude of the electromagnetic field (for the same quiver velocity) allows us to test similar conditions met in the real experiment in which high pump frequency is used.

code only for the relatively low-frequency plasma wave.

However, when the pump driver frequencies are high, we expect that the interaction is much weaker for the same quiver velocity. Thus, decreasing the amplitude of the electromagnetic field allows us to test similar conditions met in real experiment when high pump frequency $\left(\omega_{0} \approx 30 \omega_{p}\right)$ are used. Keeping the same pump frequency $\omega_{0}=2.60 \omega_{p}$ to make the comparison between both Vlasov-Maxwell and Vlasov-Hilbert codes, we now take a smaller value of the electromagnetic field of $e E_{0} / m \omega_{p} c=0.188$. As previously, Fig. 4 summarize the simulation results: we have plotted the different mean action density evolution versus time. Figure 4(a) [and 4(c)] correspond to the Vlasov-Maxwell simulation, while Fig. 4(b) [and 4(d)] have been obtained using the Vlasov-Hilbert code. Again, as expected for the electromagnetic quantities [shown in Fig. 4(a) and Fig. 4(b)], good agreement is obtained. The chief difference met previously, in Figs. 3(c) and 3(d) for a higher value of $e E_{0} / m \omega_{p} c=0.32$, in the relative amounts of $N_{0}$ and $N_{e}$ is now strongly reduced.

\section{NUMERICAL SIMULATION OF HIGH-FREQUENCY ELECTROMAGNETIC BEATWAVE}

In this section, we make a detailed presentation of the numerical experiment concerning the beat of two very highfrequency laser beams, in order to illustrate the real possibilities of the HV model. We assume a simplified system in which the electromagnetic field spectrum is composed of the pump, idler (Stokes) components, the other components being negligible. The plasma wave has correspondingly only one main Fourier component. Thus the system can be adequately described as a three-wave system. We have not included the anti-Stokes wave and cascade process to lowerfrequency waves, which start to be important at later stages of the instability. We simulate a beatwave process using a pump frequency close to $\omega_{0}=30.01 \omega_{p}$ and an idler frequency of $\omega_{s}=28.96 \omega_{p}$, close to the UCLA experiment, ${ }^{1}$ in which the experimental ratio of driver frequency to plasma frequency is $\omega_{0} / \omega_{p} \approx 33$. (For economy in this demonstration, our pump and idler rise times are chosen to be faster than the 
TABLE II. Numerical parameters used in the Hilbert-Vlasov simulations of the high-frequency electromagnetic beatwave of Sec. V A.

\begin{tabular}{llll}
\hline \hline & $\begin{array}{c}\text { Pump } \\
\text { wave }(o)\end{array}$ & $\begin{array}{c}\text { Stokes } \\
\text { wave }(s)\end{array}$ & $\begin{array}{c}\text { Plasma } \\
\text { wave }(e)\end{array}$ \\
\hline Frequency $\omega / \omega_{p}$ & 30.01 & 28.96 & 1.04723 \\
Wave number $k c / \omega_{p}$ & 30 & 28.952 & 1.04790 \\
Group velocity $\nu_{g} / c$ & 0.99966 & 0.99972 & 0.088 \\
Entry quiver $\nu_{\mathrm{osc}(0, s)} / c$ & 0.07068 & 0.07325 & 0.99936 \\
Phase velocity $\nu_{\varphi} / c$ & & & \\
Entry quiver $p_{\mathrm{osc}(0, s)} / m c$ & 0.07086 & 0.07344 & 27.95 \\
Phase momentum $p_{\varphi} / m c$ & 0.2094 & 0.2170 & 5.996 \\
Wavelength $\lambda \omega_{p} / c$ & & \\
\hline \hline
\end{tabular}

experiment.) Here the code is in a frequency ratio regime so extreme that no comparison with the Maxwell-Vlasov code is possible, due to the fact that the computer burden is prohibitive. We will discuss, for comparison with our earlier work, the same hot plasma just discussed and then a plasma much closer to the much colder UCLA experiment, first without and then with an injected electron beam.

\section{A. High-frequency beatwave simulation without beam injection}

We study the beat of two high-frequency driver waves in the parameter range $\left(\omega / \omega_{p} \approx 30\right)$, where the phase velocity of the created plasma wave is very close to the light velocity $c$ (in fact, we have $\nu_{\varphi} / c=0.99936$ ). The corresponding energy of the fast electrons is highly relativistic $\left(\gamma_{\varphi}=27.97\right)$. The numerical parameters are presented in Table II. We choose a plasma slab of length $L=600 \mathrm{c} / \omega_{p}$, which corresponds to $L=2864 \lambda_{0}$ or $L \approx 100 \lambda_{e}$. As before, for the numerical experiments presented in Sec. IV, the plasma wave was chosen with two electron temperature components: the majority (95\%) component with a $15 \mathrm{keV}$ temperature and a minority component (5\%) at $100 \mathrm{keV}$ to enhance the acceleration process. We keep here the same rise time profile of the type $\sin ^{2}\left(\pi t \omega_{p} / 100\right)$ for both laser beams. Furthermore, the laser beams are injected into plasma with equal amplitudes of $e E_{0} / m \omega_{p} c=3$.

We present in Fig. 5 the numerical results obtained from the HV code at time $t \omega_{p}=400$. The curves show the envelope of the pump wave [Fig. 5(a)] and its corresponding idler wave in Fig. 5(b) in action units, while the longitudinal electric field is illustrated in Fig. 5(c) (in $m \omega_{p} c / e$ units) and its corresponding envelope in Fig. 5(d) in the action units. Since the frequencies are so high, the interaction is much weaker for the same quiver velocities, the differences between the HV and the Maxwell-Vlasov results are now much closer than those presented in Figs. 2 and 3. Note that there is already some pump depletion corresponding to idler wave buildup cycles, together with the growth of the plasma wave. The curves exhibit the onset of the well-known three-wave oscillatory behavior in which the energy is transferred back and forth between the pump, idler, and plasma waves. The beat of both driver electromagnetic waves is clearly occurring, leading to a weak depletion of the idler wave around the value $x \omega_{p} / c=130$. At that time the corresponding plasma wave reaches its saturation level, leading to a wave packet (a

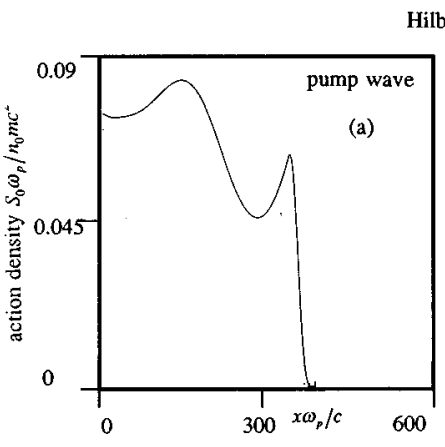

Hilbert-Vlasov

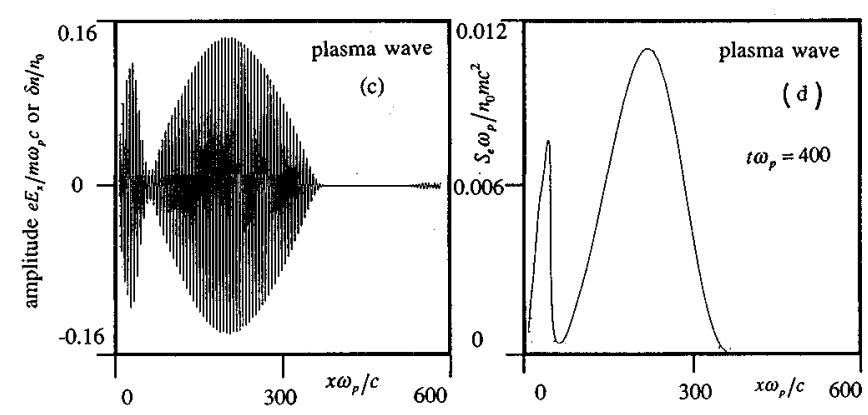

FIG. 5. We present here, at time $t \omega_{p}=400$, the numerical results obtained from the Hilbert-Vlasov code in the case of the study of high-frequency beatwave with a ratio of driver frequency to plasma frequency close to $\omega_{0} / \omega_{p} \approx 30$. The normalized driver and idler action densities, together with the oscillating electric field (in normalized $m \omega_{p} c / e$ unit for the electric field) and its action density are plotted at two different times.

"soliton-like structure"), which is well behaved with a smooth envelope. The first left-hand structure observed in the electric field is probably due to a too-rapid growth of driver laser intensity in the plasma.

In obtaining significant electron energy from plasma waves, a balance must be struck between operating with such heavy damping or plasma wave loading that wave growth is stifled and such light loading that few electrons are accelerated. In the present study, the situation is different because the electron plasma wave has a large phase velocity very close to $c$. Second, the relativistic variation of the electron mass that induces a nonlinear detuning can also limit the growth of the plasma wave. To check this point, it is interesting to compare the saturation level of the electric field, with the prediction given by the relativistic saturation limit of Rosenbluth and Liu, ${ }^{10}$ which was obtained by assuming a constant amplitude for the driver waves. A little algebra yields the Rosenbluth-Liu ${ }^{10}$ amplitude of the electric field:

$$
\frac{e E_{\mathrm{RL}}}{m \omega_{p} c}=\left(\frac{k_{e} c}{\omega_{p}}\right)^{-1}\left(\frac{8}{3} \frac{c^{2} \nu_{\mathrm{osc} 0} \nu_{\mathrm{osc} S}}{\nu_{\varphi}^{4}}\right)^{1 / 3} .
$$

With the values given by Table II, the saturation amplitude is then $e E_{\mathrm{RL}} / m \omega_{p} c=0.229$, while the code gives a maximum electric field of $0.145 m \omega_{p} c / e$ at time $t \omega_{p}=400$, i.e. a ratio of 0.633 . In density action units, we obtain $S_{e} \omega_{p} / n_{0} m c^{2}=0.0275$ for the Rosenbluth-Liu saturation limit, while the numerical value is close to 0.011 , as can be seen in Fig. 5(d). Comparing these numerical results with the case of the coupled mode model shown in Fig. 6 (without damping term) shows similar typical phenomena. It is clear that the general behavior is well reproduced, except for the 


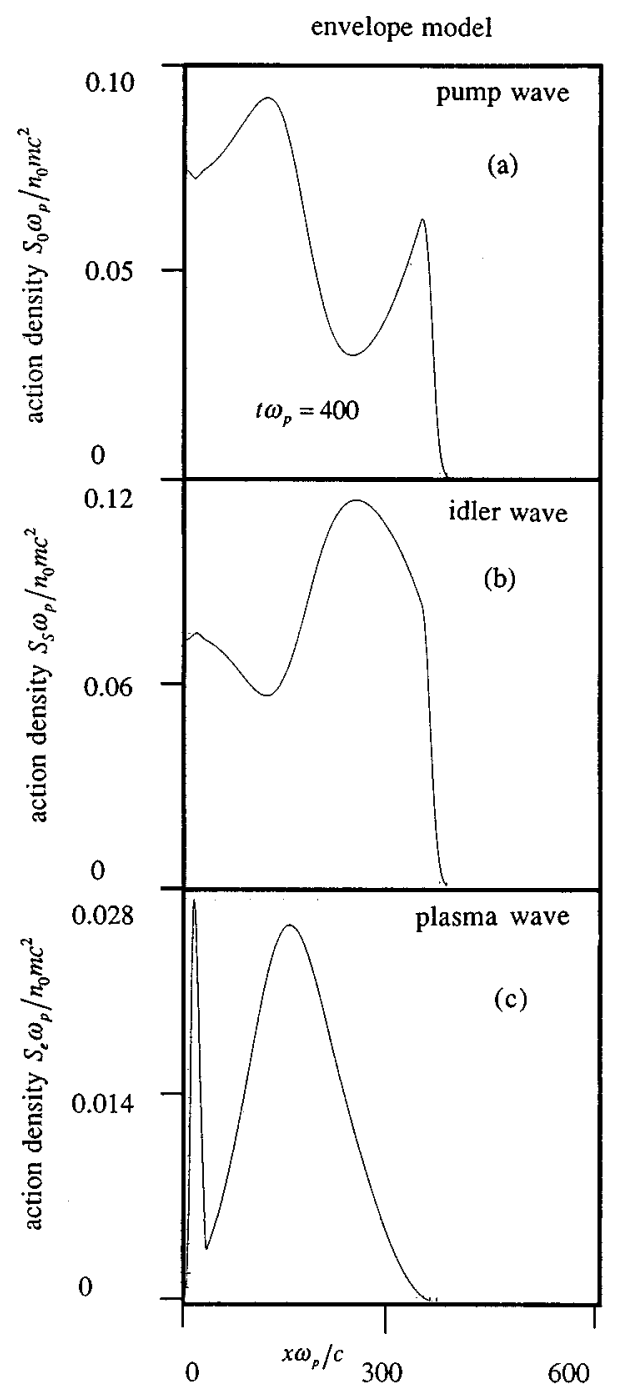

FIG. 6. Numerical results for the same parameters as Fig. 5, obtained from coupled mode model without a damping term, indicate similar behavior compared with Fig. 5. We have plotted the envelopes of the pump (a), idler (b), and the corresponding plasma (c) wave in density action units.

amplitude of the electric field (which approaches the Rosenbluth-Liu amplitude limit) due to the fact that the wave-particle interactions are not correctly taken into account in the envelope model. The differences between electric fields computed by each model are principally due here to the high value of the hot plasma temperature $T_{e}=100 \mathrm{keV}$, giving rise to a strong Landau damping level. In the threewave envelope model, the value of this damping term may be adjusted, so that the maximum value of the electric field of the plasma wave would correspond to the value obtained from the kinetic simulation. However, as mentioned in Ref. 5, for waves varying in both space and time, an ad hoc damping term chosen after the fact is not a substitute for a better understanding of particle trapping loading of the plasma wave. Finally, rather than an analysis of the simulation results in term of wave coupling using a three-mode envelope model, we have extended the study of integrated action diagnostics.

A detailed examination of the phase space seems to indicate that although the peak electric field amplitude of Fig.
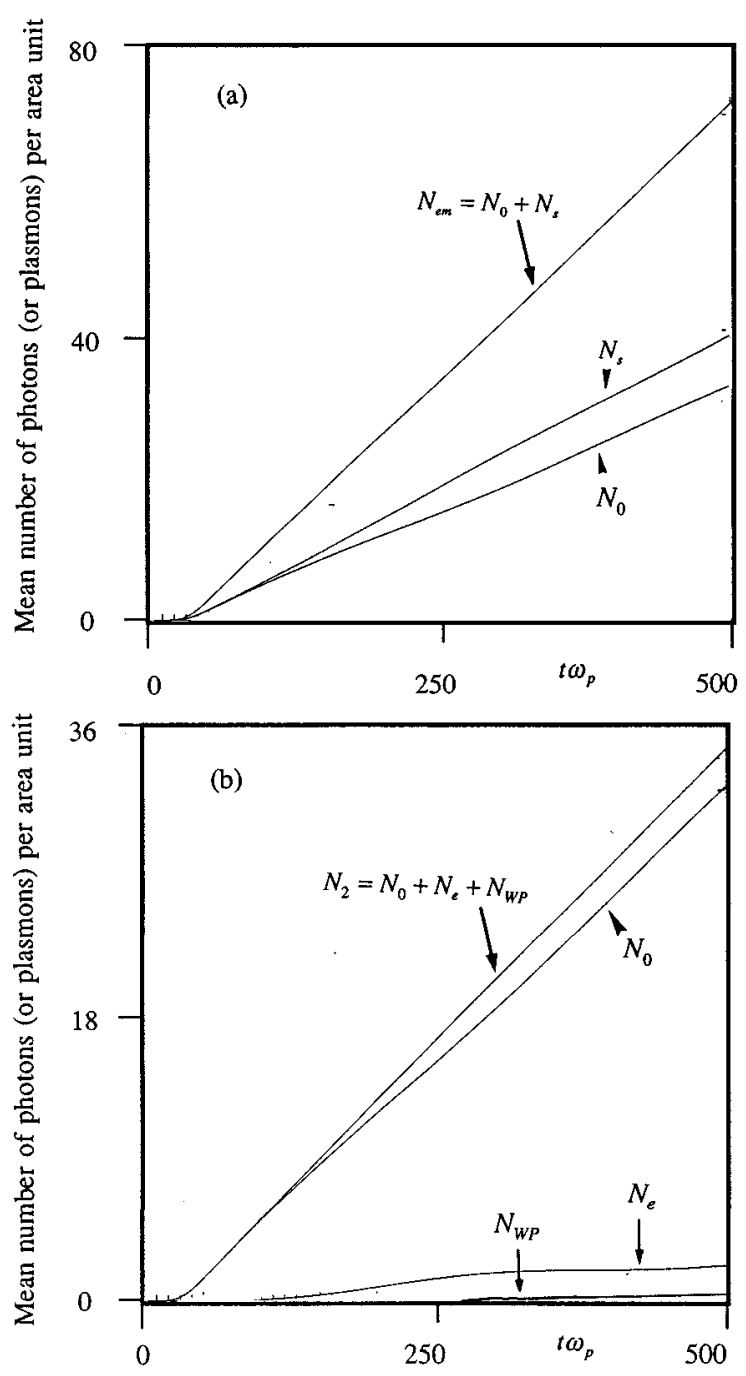

FIG. 7. Time evolution of the normalized mean number of pump photons $N_{0}$ (per area unit), of idler photons $N_{s}$ and the corresponding mean number of plasmons $N_{e}$ and their respective sums using the Hilbert-Vlasov model in the case of the ratio of the driver frequency to the plasma frequency is $\omega_{0} / \omega_{p} \approx 30$ : (a) $N_{\mathrm{em}}=N_{0}+N_{s}$, (b) $N_{2}=N_{0}+N_{e}+N_{\mathrm{WP}}$. Note that in this case the mean number of plasmons due to wave-particle interaction is negligible.

5 is comparable to that of Fig. 2, the much higher plasma wave phase momentum $\left(p_{\varphi} / m c=21.95\right.$ vs 1.8$)$ means that only a weak level of action transfer to electrons is found. This regime corresponding to a negligible particle loading effect allows us to confirm the accuracy of our HilbertVlasov model by verifying the temporal evolution of the number of photons and plasmons in the system. Figure 7(a) displays the variation with time of the mean number of pump photons (per area unit) $N_{0}, N_{s}$ (for the idler wave) and their sum $N_{\mathrm{em}}=N_{0}+N_{s}$. For $t \omega_{p} \geqslant 50$ (corresponding to the progressive increase of both driver waves), the $N_{\text {em }}$ curve is then a straight line with a slope close to $F_{\text {num }} \hbar \omega_{p} / n_{0} m c^{3}=0.1530$; this value agrees well with the expected value $\left(F_{0}+F_{s}\right) \hbar \omega_{p} / n_{0} m c^{3}=0.1526$, which measures the total photon flux continuously injected into the plasma. The time behavior of the other action densities [given by Eqs. (23) and (24)] from the HV simulation are shown in Fig. 7(b), together with the relevant action sum including the mean number of plasmons due to wave-particle interactions. 
We have thus plotted the following quantities $N_{0}, N_{e}, N_{\mathrm{WP}}$ (with the same notations as previously indicated), and finally their sum $N_{2}=N_{0}+N_{e}+N_{\text {WP }}$ in Fig. 7(b). First, the simulation results indicate a relatively low action transfer level close to $N_{\mathrm{WP}} \hbar \omega_{p} / n_{0} m c^{2} \approx 0.5$, about $1.5 \%$ of $N_{s}$. In other words, the action transfer to electron acceleration is almost negligible in the present simulation. The general behavior of action sum terms is, as expected, exhibiting straight lines with slopes of $F_{\text {num }} \hbar \omega_{p} / n_{0} m c^{3}=0.0734$ for $N_{2}$ close to the theoretical value of the pump photon flux $F_{0} \hbar \omega_{p} / n_{0} m c^{3}=0.0749$. Similar treatment of the quantity $N_{3}=-N_{s}+N_{e}+N_{\text {WP }}$ gives an evaluation of the idler wave flux of $F_{\text {num }} \hbar \omega_{p} / n_{0} m c^{3}=-0.0787$, found also in good agreement with the photon flux of the idler wave $F_{s} \hbar \omega_{p} / n_{0} m c^{3}=-0.0776$.

The simulation at temperature $T_{e}=15 \mathrm{keV}$ has shown important deviations from the results obtained by the usual envelope model, even if no electron beam was injected into the plasma, presumably due to the significant electron acceleration effects. To confirm this point, a run has been performed with a much lower cold plasma temperature component, now $T_{e}=1 \mathrm{keV}$, and no hot component at all. The evidence is provided in Fig. 8, where the longitudinal electric field $e E_{x} / m \omega_{p} c$ is shown in Fig. 8(a) and its corresponding electric action density $S_{e} \omega_{p} / n_{0} m c^{2}$ in Fig. 8(b) at time $t \omega_{p}=400$. These results are now in close agreement with those directly computed by the envelope model, plotted in Fig. 8(c), in action density units. Agreement does not deteriorate until the initial temperature is increased to the value $T_{e}=5 \mathrm{keV}$. For reasons of numerical burden (particularly when the beam injection is also to be taken into account), it is difficult to decrease the temperature low $T_{e}=1 \mathrm{keV}$ in the code in these conditions. Note the presence of a very rapid growth of the electric field at the left-hand side of the plasma box over a distance less than $5 \omega_{p} / c$. (This artifact is a reason to consider a less rapid variation of electron density at the left-hand boundary.)

\section{B. High-frequency beatwave experiment with electron beam injection}

\section{Numerical simulation with modest quiver velocities}

Another example is now given to illustrate the possibilities of the code for the study of a beatwave case relevant to the UCLA experiment with external injection of an electron beam. A series of simulations were performed in this case. As a first example, the cold plasma condition has left its previous value of $15 \mathrm{keV}$, but the hot component is replaced by an electron beam, and we have chosen a plasma length double that of Sec. V A, namely $L \omega_{p} / c=1200$. Explicitly, the initial distribution function includes an electron beam and can be written in the form

$$
\begin{aligned}
f\left(x, p_{x}, t=0\right)= & \frac{\alpha}{\sqrt{2 \pi} \nu_{\mathrm{th}}} e^{-\left(p_{x}^{2} / 2 m^{2} c^{2}\right)\left(c^{2} / \nu_{\mathrm{th}}^{2}\right)} \\
& +\frac{(1-\alpha)}{\sqrt{2 \pi} \nu_{\mathrm{th} b}} e^{-\left[\left(p_{x}-p_{b}\right)^{2} / 2 m^{2} c^{2}\right]\left(c^{2} / \nu_{\mathrm{thb}}^{2}\right)},
\end{aligned}
$$

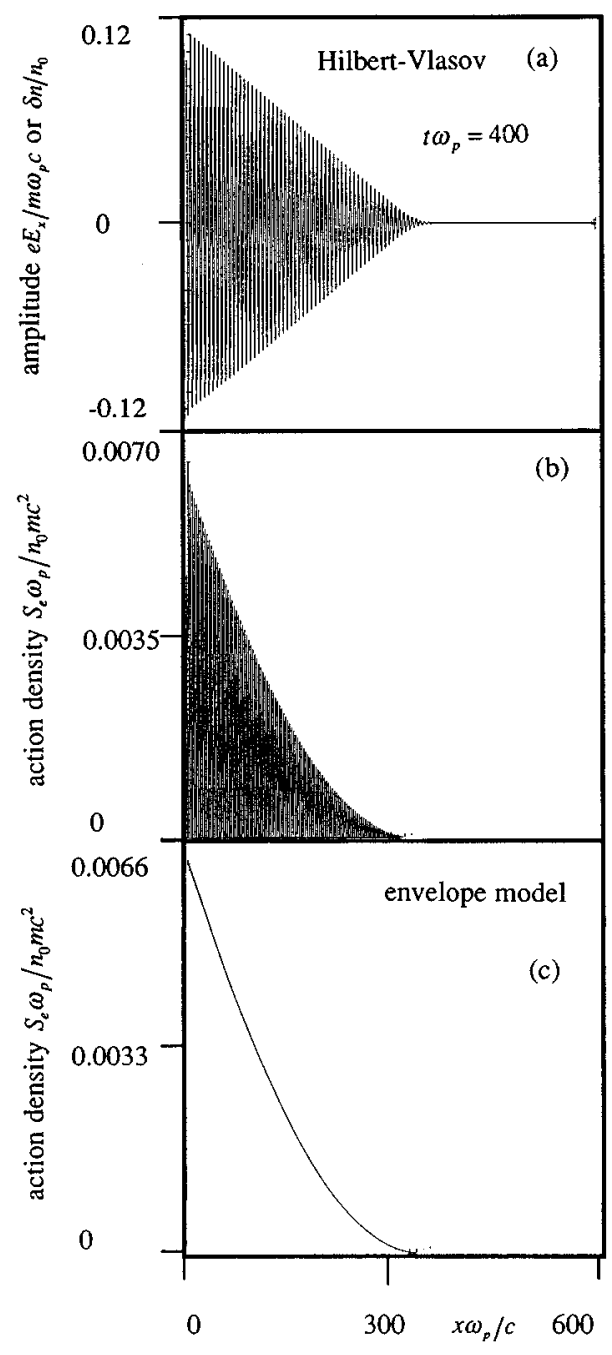

FIG. 8. A case similar to Figs. 6 and 7, except the plasma is cold $\left(T_{e}=1\right.$ $\mathrm{keV}$ ), at time $t \omega_{p}=400$ of the normalized longitudinal electric field in (a) and its corresponding action density in (b). Excellent agreement is obtained with results calculated by an envelope model plotted in (c).

where $\nu_{\text {th }}$ is the cold thermal velocity given by $\nu_{\text {th }} / c=0.1713$, corresponding to an electron bulk temperature of $15 \mathrm{keV}$ while $\nu_{\text {th } b}$ is the beam thermal velocity. We have $\nu_{\text {th } b}$ $=0.442 c$. We take $p_{b} / m c=12$ and $\alpha=0.9990$ (i.e., the relative beam density is $1-\alpha=10^{-3}$ ), corresponding to a beam injection at $6 \mathrm{MeV}$ and we have taken the maximum quiver velocities of $\nu_{\mathrm{osc} 0} / c=0.070$ and $\nu_{\mathrm{oscS}} / c=0.073$, with a rapid rise time of $\tau \omega_{p}=50$.

Figure 9 shows, at time $t \omega_{p}=600$ the pump, idler, and plasma wave action envelopes in density action units, together with the oscillating longitudinal field (given in $m \omega_{p} c / e$ units). The curves show the formation of a large wave packet for the electric field of length $300 c / \omega_{p}$ (corresponding to a length of $1400 \lambda_{0}$ or $50 \lambda_{e}$ ). Because of the low beam density, no beam-plasma instability occurs in the system. On comparing Fig. 9 with Fig. 5, there appears to be a more rapid spatial oscillation in the plasma wave envelope with the addition of the electron beam. It must be pointed out that rather sharp temporal oscillations take place near the front edge of the plasma, but remain localized into this region during evolution. More striking is the fact that the satu- 

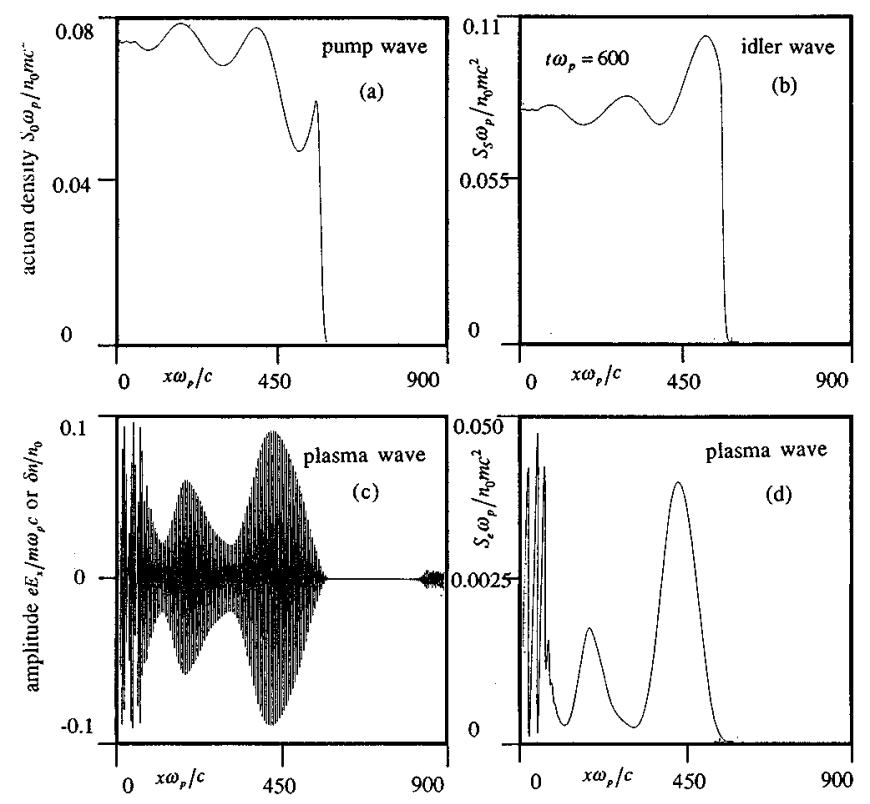

FIG. 9. The case of beam injection into the plasma relevant of a UCLA beatwave experiment with $\omega_{0} / \omega_{p}=30$. The curves show at time $t \omega_{p}=600$ the pump, idler wave envelopes in (a) and (b), respectively, together with the oscillating electric field shown in (c) and its envelope in (d).

ration level of the electric field [i.e., $e E_{\mathrm{sat}} / m \omega_{p} c=0.10$, corresponding to a density modulation of $\delta n / n_{0}$ $\left.=\left(e E_{\mathrm{sat}} / m \omega_{p} c\right)\left(k_{e} c / \omega_{p}\right) \approx 10 \%\right]$ is now weaker than the one observed in previous simulation without beam injection and to indicate increased energy transfer from the plasma wave to particles compared with Fig. 8. Further confirmation of this feature is given by the phase space representation of the distribution function. Here we give a detailed examination of such ultrahigh gradient acceleration of externally injected electrons by a laser beatwave excited relativistic electron plasma wave. Because of its very fine resolution in phase space, the Hilbert-Vlasov code is capable of resolving the finest details of the particle acceleration. As an example, Fig. 10 shows a window of length $l=115 \lambda_{0}$ (i.e., $4 \lambda_{e}$ ) moving at the linear wave phase velocity. We have used grey shading to represent the distribution function $f$ between $10^{-5}$ and $10^{-4}$ (black for $f>10^{-4}$ and white for $f<10^{-5}$ ). The solid curves superimposed in Fig. 10 represent the limiting trapped orbit, i.e. the instantaneous separatrix of particles (as computed from the electric potential given by the code). Because the window is carefully tailored to move at the phase velocity, the trapping structure, seen in Fig. 10, are quite stationary in this frame, giving a time history at the same population of trapped particles. Beam electrons are injected in strong field region so that they become accelerated to high energies. These fast electrons cannot escape the strongest field region since the upper trapping limit (which is not plotted here) reaches a maximum value of $p_{\max } / m c \approx 1000$. The maximum energy gain of accelerated electrons is due entirely to the limits in time and space of the simulation.

Incidentally, for the simulations of this section, with no hot plasma electrons, no energetic electrons were observed to be produced by the beat of two electromagnetic waves, when none were injected into plasma, in agreement with the UCLA
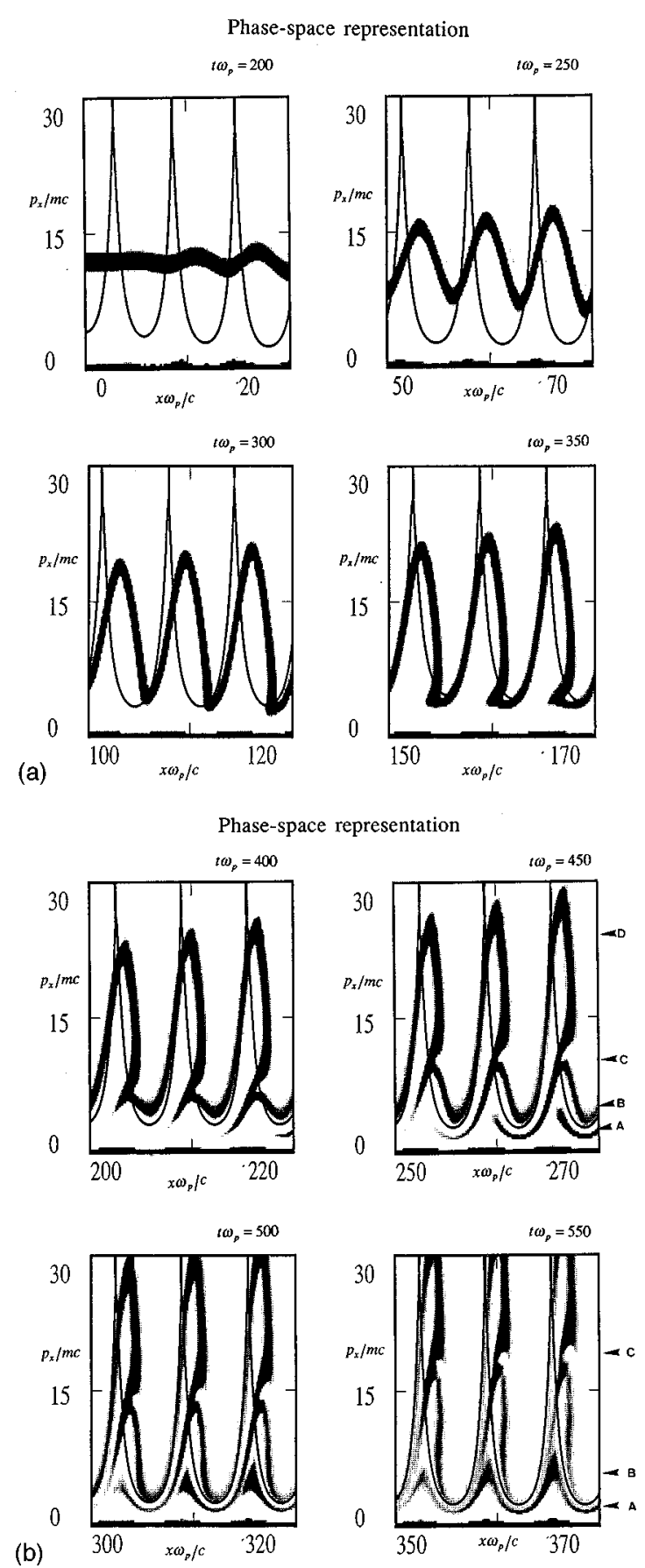

FIG. 10. Simulation of Fig. 9: phase space densities in a window of length $l=3 \lambda_{e}$ moving at the light velocity. The solid lines mark the separatrix limit between the trapped and untrapped particle orbits for the local amplitude of the plasma wave. The curves indicate clearly the strong acceleration mechanism up to momentum close to $p_{\max } / m c \approx 30$ by $t \omega_{p}=450$. At $t \omega_{p}=450$, 550 , values of momentum corresponding to specific energy spectrum features are marked. The corresponding momentum are $p_{x} / m c=1.68(\mathrm{~A})$, $p_{x} / m c=3.78(\mathrm{~B}), p_{x} / m c=9.73(\mathrm{C})$, and $p_{x} / m c=24.4$ (D) at $t \omega_{p}=450$. At later time $t \omega_{p}=550$, we have, for momentum values, $p_{x} / m c=2.04(\mathrm{~A})$, $p_{x} / m c=5.21(\mathrm{~B})$, and $p_{x} / m c=18.56$ for $(\mathrm{C})$.

experiment. Also, there is no detectable contribution to the acceleration process due to self-trapping of the background plasma electrons in excited plasma oscillations. Third, the $x-p_{x}$ distributions show clearly that some beam electrons suffer deceleration from the plasma wave (these particles have momentum close to $p_{x} / m c \approx 2-3$ ) passing slightly be- 


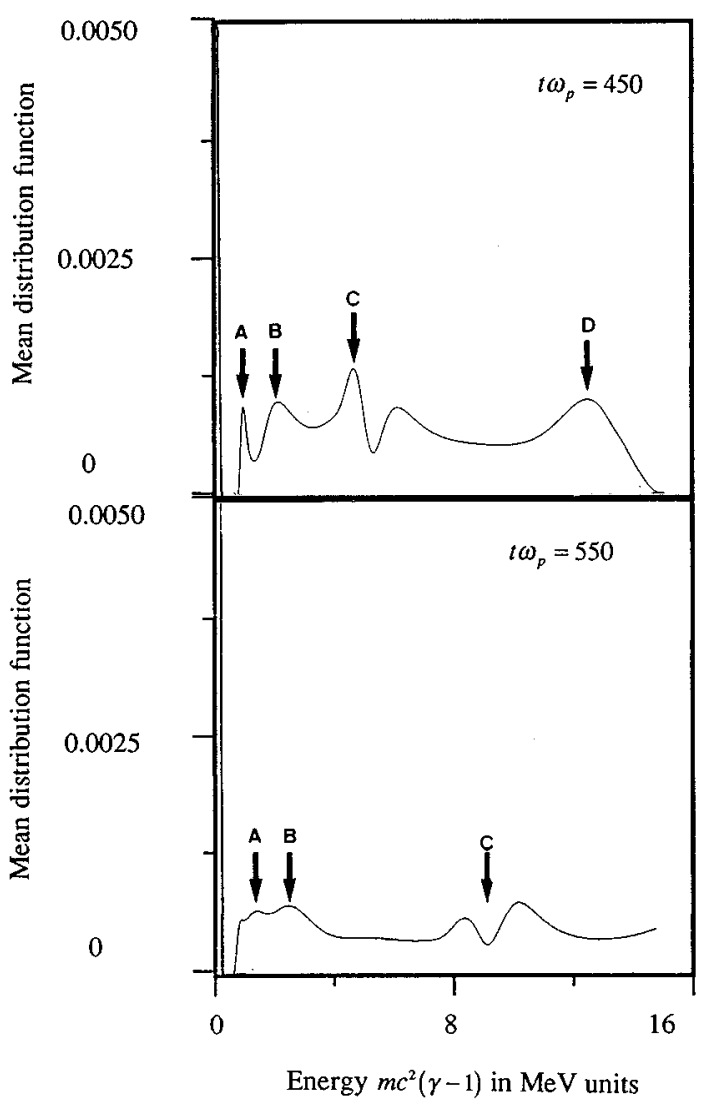

FIG. 11. Behavior of the mean distribution function (averaged over the window length) for $t \omega_{p}=450,550$ as a function of the kinetic relativistic energy. The simulation demonstrates the generation of ultrafast electrons of energies that exceed $15 \mathrm{MeV}$, leading to an ultrahigh gradient acceleration close to $0.7 \mathrm{GeV} / \mathrm{m}$. Also note the beginning of the separation at $5 \mathrm{MeV}$ into fast and slow electron beam components and the specific features corresponding to Fig. 10. Again the corresponding momentum values are $p_{x} / m c=1.68(\mathrm{~A}), p_{x} / m c=3.78(\mathrm{~B}), p_{x} / m c=9.73(\mathrm{C})$, and $p_{x} / m c=24.4(\mathrm{D})$ at $t \omega_{p}=450$. At later time $t \omega_{p}=550$, we have, for momentum values, $p_{x} / m c=2.04$ (A), $p_{x} / m c=5.21(\mathrm{~B})$, and $p_{x} / m c=18.56$ for $(\mathrm{C})$.

low the lower separatrix limit, in good agreement with adiabatic theory predictions. ${ }^{11,12}$ Finally, at time $t \omega_{p} \approx 450-500$, the wave-particle interaction becomes stronger and more complicated due to the larger number of fast electrons. More details of the particle distribution functions can be found in Fig. 10, which display $\left\langle f\left(x, p_{x}\right)\right\rangle$, at two different times; $t \omega_{p}=200$ and $t \omega_{p}=450$. The spatial average $\left\langle f\left(x, p_{x}\right)\right\rangle$ is over three wavelengths of the plasma wave, corresponding to the size of the moving window. In Fig. 11, the momentum scale is replaced by the relativistic kinetic energy in $\mathrm{MeV}$ units. The simulation demonstrates the generation of ultrafast electrons with $\gamma \geqslant 30$ corresponding to total energies that exceed $15 \mathrm{MeV}$. Therefore some electrons gained at least 10 $\mathrm{MeV}$ in traversing the roughly $1400 \lambda_{0}$-long plasma wave (corresponding to an interaction length of $1.43 \mathrm{~cm}$ for a $\mathrm{CO}_{2}$ laser of wavelength $\lambda_{0}=10.275 \mu \mathrm{m}$ ), implying an acceleration gradient of more $0.7 \mathrm{GeV} / \mathrm{m}$, which is in semiquantitative agreement to values observed in the UCLA experiment. ${ }^{1}$

We now examine the time evolution of Manley-Rowe actions. Figure 12(a) shows the mean number of pump photons $N_{0}$, and idler photons $N_{s}$, and their mutual sum $N_{\mathrm{em}}=N_{0}+N_{s}$. As expected, the total mean photon number
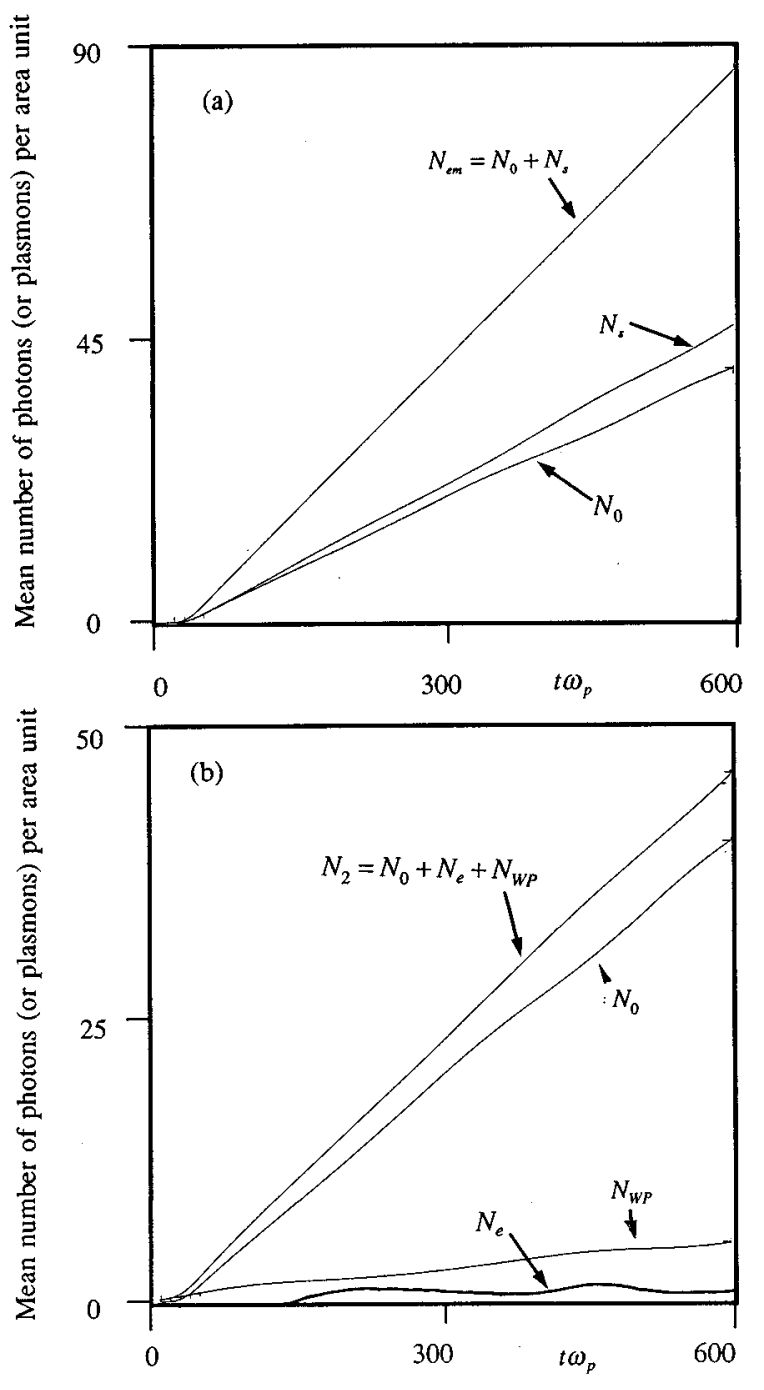

FIG. 12. Display of the time evolution of the different mean number of photons or plasmons per area unit and their respectives sums: (a) $N_{\mathrm{em}}=N_{0}+N_{s}$, (b) $N_{2}=N_{0}+N_{e}+N_{\mathrm{WP}}$ (note that $N_{\mathrm{WP}} \gg N_{e}$ ). These numerical results are relevant to the study of a beam injection into the plasma for high ratio of driver frequency to plasma frequency $\omega_{0} / \omega_{p} \approx 30$.

sum display exhibits a straight line with a slope evaluated to be 0.1520 (see Table II), which is in good agreement with the theoretical value of 0.1526 of the total photon flux given from $F_{0}+F_{s}$. Since the plasma wave interacts nonlinearly with both driver waves as well as with the trapped and nearly trapped electrons, the way to isolate the interaction between the plasma wave interaction with the trapped electrons is to consider the action sum of pump wave $N_{0}$, plasma wave $N_{e}$, and "the transferred action" due to the wave-particle interactions we have called $N_{\mathrm{WP}}$. We have computed the quantity $N_{2}=N_{0}+N_{e}+N_{\mathrm{WP}}$ (note that $2 N_{\mathrm{aS}}$ is negligible here), and the result is shown in Fig. 12(b). The temporal variations give the pump photon flux. Numerical values obtained by the Hilbert-Vlasov code are in good agreement with the theoretical ones (a comparison is shown in Table III), which allows us to check the accuracy of the action transfer evaluated through the data of the relativistic kinetic energy of particles above the lower separatrix. This behavior also makes it clear that a simple coupled-mode theory for the description of the 
TABLE III. Comparison of numerical and analytic results of normalized photon flux obtained from the data of the mean number of photons and plasmons in the box simulation (Manley-Rowe action evolution).

\begin{tabular}{lcc}
\hline \hline & $\begin{array}{c}\text { Normalized } \\
\text { theoretical } \\
\text { photon flux } \\
F_{i} \hbar \omega_{p} / n_{0} m c^{3}\end{array}$ & $\begin{array}{c}\text { Normalized } \\
\text { numerical } \\
\text { value } \\
F_{i} \hbar \omega_{p} / n_{0} m c^{3}\end{array}$ \\
\hline$N_{e m}=N_{0}+N_{s}$; Eq. (17) & $F_{0}+F_{s}=0.1526$ & 0.1520 \\
$N_{2}=N_{0}+N_{e}+N_{\mathrm{WP}}$ Eq. (23) & $F_{0}=0.0749$ & 0.079 \\
$N_{3}=-N_{s}+N_{e}+N_{\mathrm{WP}}$ Eq. (22) & $F_{s}=0.0776$ & 0.072 \\
\hline \hline
\end{tabular}

plasma wave cannot be adequate if energy transfer (due to the beam injection) to trapped particles is significant and that electron trapping effect must be correctly included.

\section{Strong particle acceleration process}

A Vlasov-Hilbert simulation of the beatwave accelerator is performed with parameters close to those of the UCLA experiment, in which a two-frequency $\mathrm{CO}_{2}$ laser has been used with wavelength $\lambda_{0}=10.275 \mu \mathrm{m}$ and $\lambda_{s}=10.591 \mu \mathrm{m}$, corresponding to values of electromagnetic frequencies normalized to the plasma frequency close to $\omega_{0}=33 \omega_{p}$ and $\omega_{s}=31.991 \omega_{p}$. The plasma temperature is taken to $T_{e}=3$ $\mathrm{keV}$ (chosen in a region wherein the Landau damping can be considered as negligible). The plasma wave phase velocity is then equal to $\nu_{\varphi} / c \approx 0.99952$, which corresponds to a phase momentum of $p_{\varphi} / m c \approx 32.314$. The acceleration mechanism is then diagnosed by injecting a beam of $2 \pm 0.10 \mathrm{MeV}$ electrons with $\alpha=0.999$ (so $1-\alpha=n_{b} / n_{p}=10^{-3}$ ). The quiver velocities of the laser have been determined using a peak intensity of $5 \times 10^{14} \mathrm{~W} / \mathrm{cm}^{2}$, which gives the maximum values of $\nu_{\mathrm{osc} 0} / c=0.2$ and $\nu_{\mathrm{osc} S} / c=0.206$ and the rise time is chosen to $\tau=600 \omega_{p}^{-1}$ corresponding to $120 \mathrm{ps}$ using the $\sin ^{2}(\pi t / 2 \tau)$ profile electromagnetic field onset. The plasma length is close to $L \omega_{p} / c=900 \approx 145 \lambda_{e}$, where $\lambda_{e}=2 \pi / k_{e} \approx 6.224 \omega_{p} / c$. The plasma wave number is then $k_{e} \lambda_{\mathrm{De}}=0.077$. The simulation has been performed using 6144 points in the $x$ direction and 2048 points in the momentum direction (for $-4 \leqslant p_{x} / m c \leqslant 60$ ). The numerical parameter concerning electromagnetic and plasma waves are given in Table IV). Figure 13 show the corresponding results at time $t \omega_{p}=500$ when the laser intensity almost reached its maximum value. The curves exhibit the spatial behavior of the pump wave [in Fig. 13(a)] and the idler wave [in Fig. $13(\mathrm{~b})]$ in action units, and the corresponding driven longitudinal plasma wave is plotted in Fig. 13(c) in $m \omega_{p} c / e$ units. At that time the normalized electric field saturates at level

TABLE IV. Numerical parameters relevant to the beatwave UCLA experiment with electron beam injection.

\begin{tabular}{lccc}
\hline \hline & \multicolumn{1}{c}{$\begin{array}{c}\text { Pump } \\
\text { wave }(o)\end{array}$} & $\begin{array}{c}\text { Idler Stokes } \\
\text { wave }(s)\end{array}$ & $\begin{array}{c}\text { Plasma } \\
\text { wave }(e)\end{array}$ \\
\hline $\begin{array}{l}\text { Frequency } \omega / \omega_{p} \\
\text { Wave number } k c / \omega_{p}\end{array}$ & 33.985 & 31.991 & 1.0089 \\
$\begin{array}{l}\text { Quiver or phase } \\
\text { momentum } p / m c\end{array}$ & 0.200 (quiver) & 0.206 (quiver) & 32.313 (phase) \\
\hline \hline
\end{tabular}

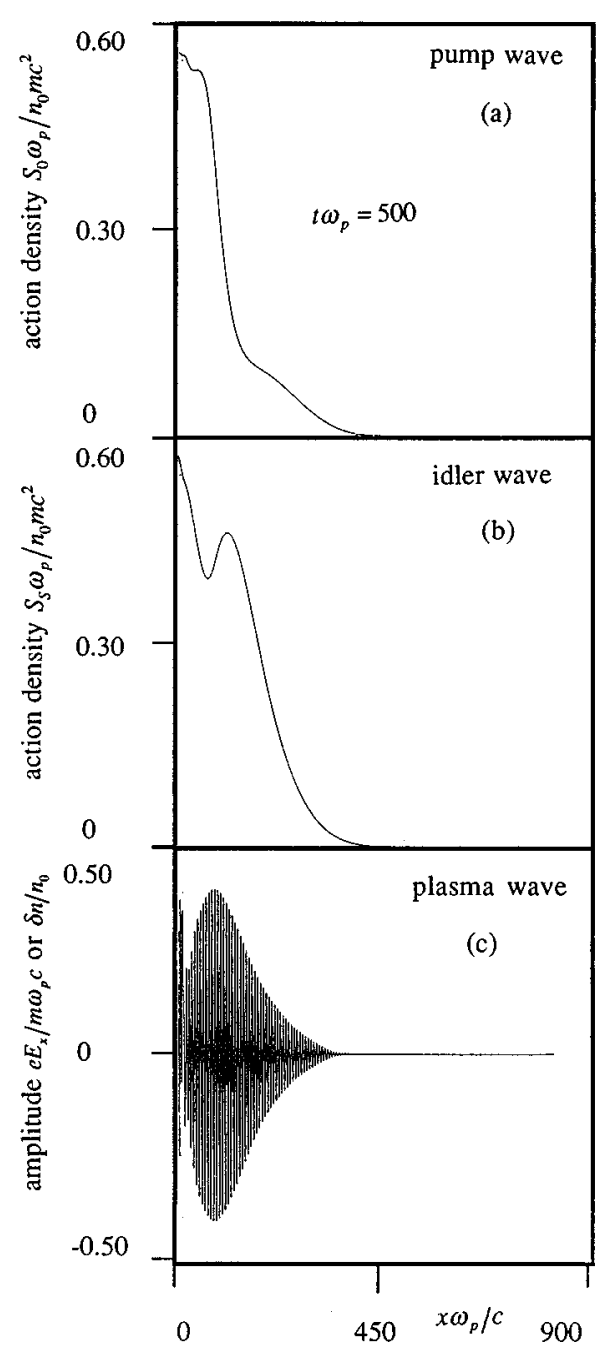

FIG. 13. Slow onset of driver and idler, with an injected beam. Plots of the pump wave in (a), idler wave in (b), and of the longitudinal electric field in (c), versus $x \omega_{p} / c$ at time $t \omega_{p}=500$. The plasma temperature is $T_{e}=3 \mathrm{keV}$ and the rise time of the laser is chosen to $\tau \omega_{p}=600$ using a $\sin ^{2}(\pi t / 2 \tau)$ profile. Note the density modulation reaches $40 \%$.

$e E_{\text {sat }} / m \omega_{p} c \approx 0.41$. This suggests an amplitude density of $\delta n / n_{0} \approx 41 \%$. The corresponding value of the RosenbluthLiu amplitude of the electric field is then in that simulation of order $e E_{\mathrm{RL}} / m \omega_{p} c=0.475$. Furthermore, Fig. 14 shows the $x \omega_{p} / c$ vs $p_{x} / m c$ phase space behavior in a window moving at the phase velocity of the plasma wave. (Some of the electron beam initially injected at energy of $2 \mathrm{MeV}$, is accelerated to the simulation boundary at $p_{x \max } / m c=60$, where the $p_{x}$ boundary condition dictates $f=0$. The loss of the beam electrons at $p_{x}=p_{x \text { max }}$ boundary changed the results by less than $2 \%$ relative to a case in which $p_{x \max }$ is set higher.) Evidence of the acceleration level is provided by the plot of the window-averaged space distribution function $\left\langle f\left(x, p_{x}\right)\right\rangle$ versus the relativistic kinetic energy $m c^{2}(\gamma-1)$ given in $\mathrm{MeV}$ units, represented in Fig. 15 at two different times during evolution $t \omega_{p}=500$ and $t \omega_{p}=660$. We can therefore take the effective acceleration length as $200 \omega_{p} / c \approx 1050 \lambda_{0}$, which corresponds to a length of $1.08 \mathrm{~cm}$ for the laser wavelength of $\lambda_{0} \approx 10.275 \mu \mathrm{m}$. Combining this with the numerically measured energy gain of $28 \mathrm{MeV}$ implies a peak accelerating 

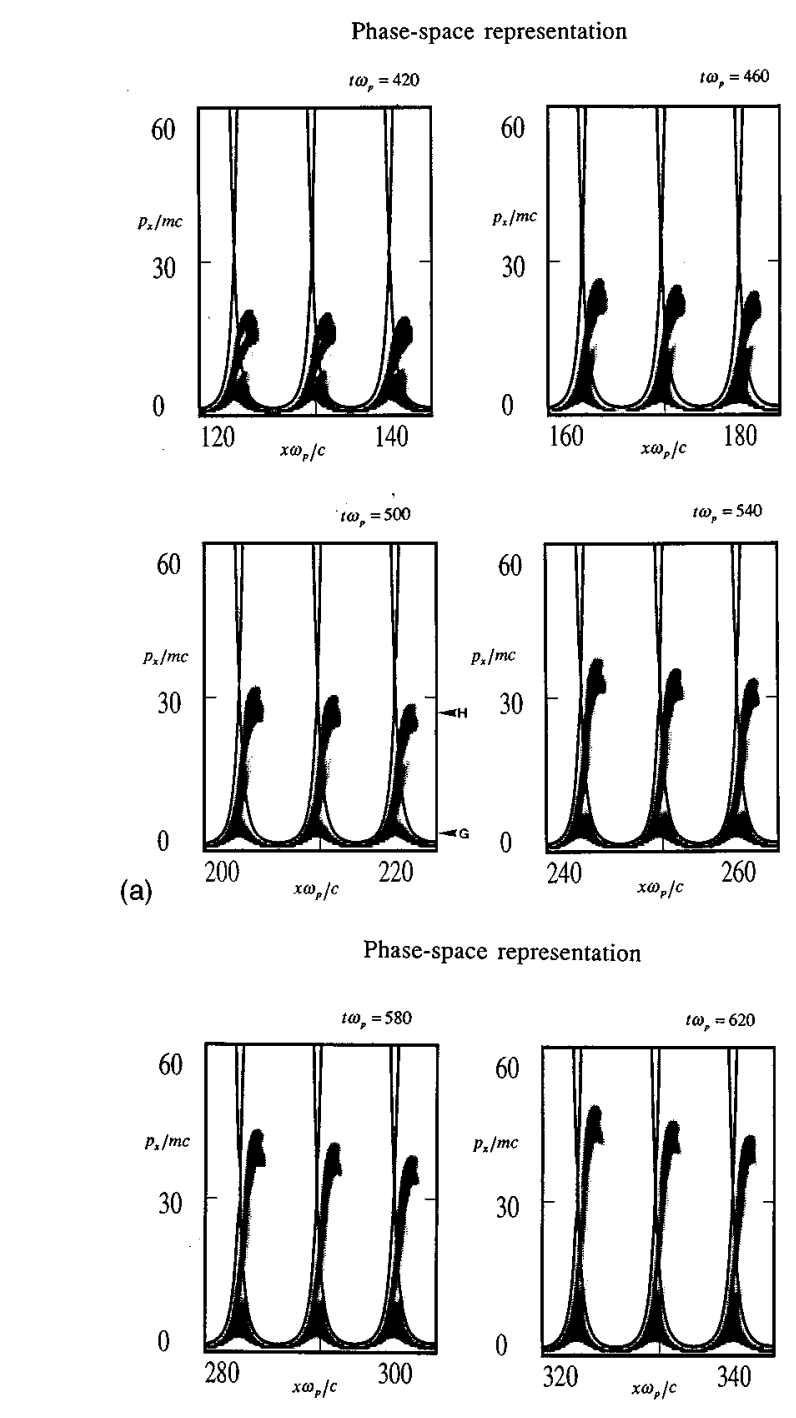

ace representation
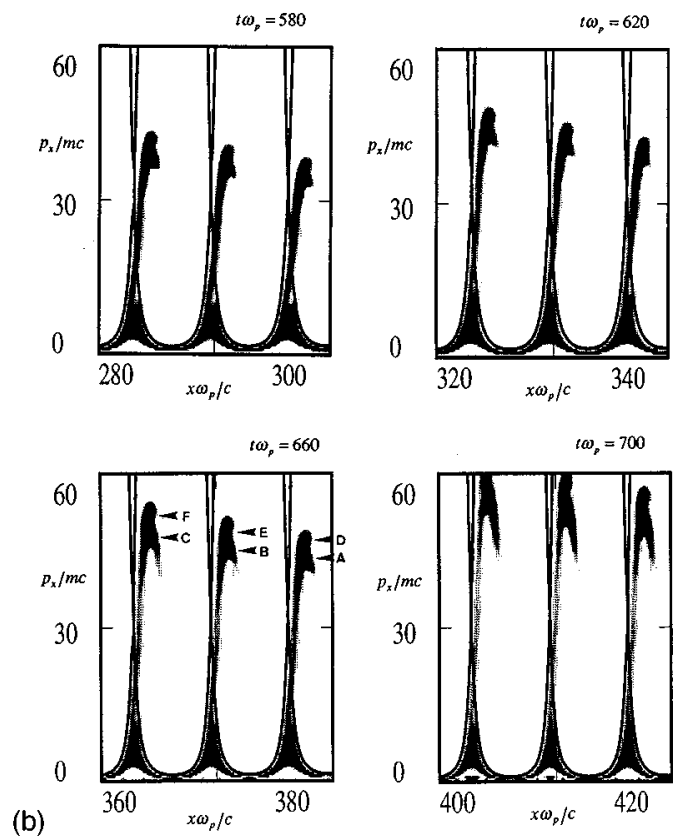

FIG. 14. For the case of Fig. 13, phase space representation of a window moving at the plasma phase velocity showing a strong acceleration process until $p_{x} / m c \approx 60$. Note the double loop structure formed by $t \omega_{p}=420$. Also note the slower structure untrapped component below the separatrix. In frames $t \omega_{p}=420,660$ features $[(\mathrm{A})-(\mathrm{F}),(\mathrm{G}),(\mathrm{H})]$ are marked for comparison with energy features of Fig. 15. The corresponding momentum values are $p_{x} / m c=42.5(\mathrm{~A}), p_{x} / m c=45.0(\mathrm{~B}), p_{x} / m c=46.9(\mathrm{C})$ and $(\mathrm{D}), p_{x} / m c=49.4$ $(\mathrm{E})$, and $p_{x} / m c=51.9(\mathrm{~F})$ at $t \omega_{p}=660$. At previous time $t \omega_{p}=500$, we have for momentum values, $p / m c=2.9(\mathrm{G})$, and $p_{x} / m c=26.4(\mathrm{H})$.

gradient of $2.6 \mathrm{GeV} / \mathrm{m}$, in reasonable agreement with values experimentally measured in the UCLA experiment. Here the peak wave amplitude is, however, higher, close to $40 \%$, which can be due the high values of the quiver velocities.

Due to the extraordinary resolution in phase space af-

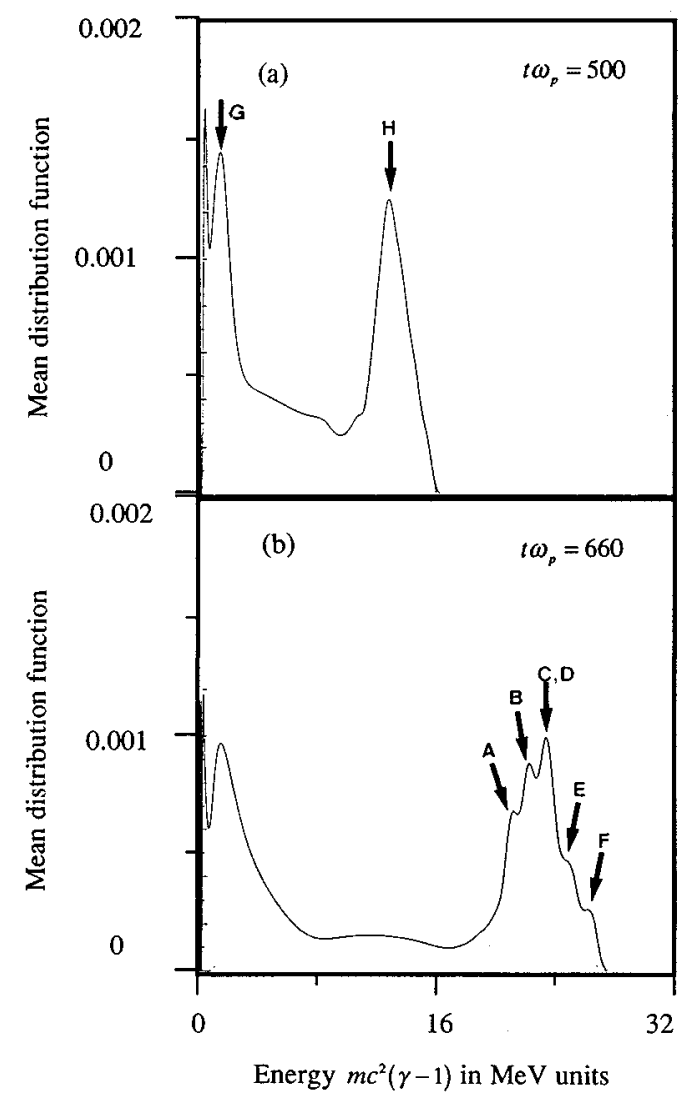

FIG. 15. Display of the mean distribution function averaged over the window length versus $m c^{2}(\gamma-1)$, showing the particle acceleration up to 28 $\mathrm{MeV}$ (which implies a peak accelerating gradient of $2.6 \mathrm{GeV} / \mathrm{m}$ ) and the separation into fast and slow components. Also note the local peaks [(A)$(\mathrm{F}),(\mathrm{G}),(\mathrm{H})]$ linked to features in Fig. 14.

forded by the Vlasov code, one can begin to understand much of the details of the interaction, as shown in Figs. 14 and 15. In keeping with the discussion on Figs. 10 and 11, one sees a further and more extreme development of the separation of the electrons into those that continue to be accelerated and those that remain untrapped below the separatrix. By considering only a three-wavelength box moving with the wave, we can account in detail for all features of the energy distribution of Fig. 15. Looking back to the earliest frame in Fig. 14, we see that there has already formed a double stream trapping structure in phase space, and it is this that accounts for the five local high-energy maxima in Fig. 15(b). [Two of the six loops in phase (three wavelengths with double structure) happen to have the same energy. The correspondence is indicated by the letters in Fig. 14 for frames $t \omega_{p}=500,600$, and in Fig. 15(b).] With such details available, one can hope to be able to study how to improve the acceleration efficiency by reducing the number of untrapped and unaccelerated electrons.

As done for the previous cases, the time behavior of the mean number of pump photons $N_{0}$ and idler photons $N_{s}$ and their mutual sum $N_{\mathrm{em}}=N_{0}+N_{s}$ are plotted in Fig. 16(a). Because the rise time of laser is no longer a small part of the run time, we must take into account the rise time profile of quiver velocities into Eq. (17), which yields for $0 \leqslant t \leqslant \tau$, 


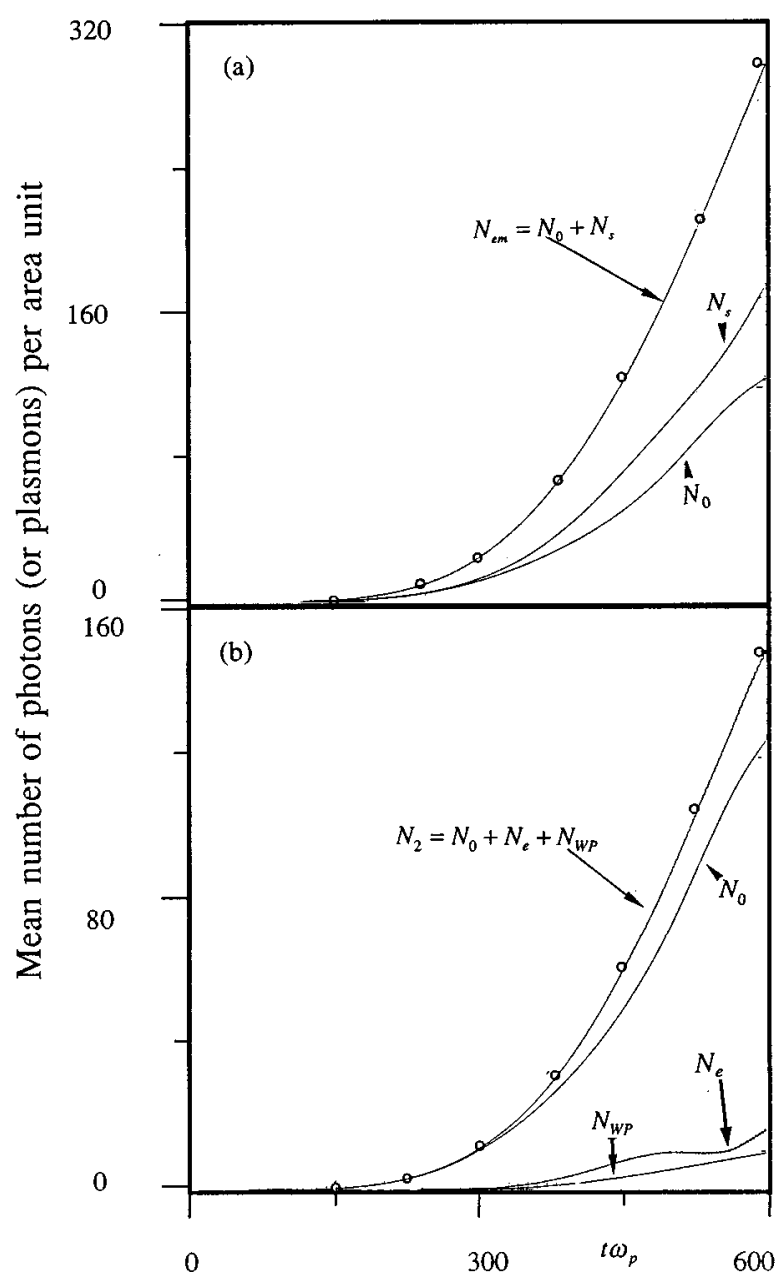

FIG. 16. From simulation of Figs. 13, 14, and 15: (a) Plots of the mean number of pump $N_{0}$, idler $N_{s}$ photons, and the corresponding sum $N_{\mathrm{em}}$, together with theoretical values versus $t \omega_{p}$. (b) Plots of the mean number of pump photons $N_{0}$, electric field plasmons $N_{e}$, wave-particle plasmons due to acceleration process $N_{\mathrm{WP}}$, and the corresponding sum $N_{2}=N_{0}+N_{e}+N_{\mathrm{WP}}$, together with theoretical values versus $t \omega_{p}$.

$$
\frac{d}{d t}\left(N_{0}+N_{s}\right)=\left(F_{0}+F_{s}\right) \sin ^{4}(\pi t / 2 \tau)
$$

It is convenient to work in terms of normalized flux $F_{i} \hbar \omega_{p} / n_{0} m c^{3}=\nu_{g} S_{i} \omega_{p} / n_{0} m c^{3}(i=0, s)$. In these variables the pump photon flux is then $F_{0} \hbar \omega_{p} / n_{0} m c^{3} \approx 0.659$ and the idler photon flux is $F_{s} \hbar \omega_{p} / n_{0} m c^{3} \approx 0.680$. Integrating Eq. (27) gives

$$
\frac{N_{0}+N_{s}}{n_{0} c}=\left(F_{0}+F_{s}\right) \frac{\hbar \omega_{p}}{n_{0} m c^{3}} G\left(t \omega_{p}\right),
$$

with $\quad G\left(t \omega_{p}\right)=\frac{3}{8} t \omega_{p}+\left(\tau \omega_{p} / 16 \pi\right) \sin (2 \pi t / \tau)-\left(\tau \omega_{p} / 2 \pi\right)$ $\times \sin (\pi t / \tau)\left[\right.$ so $\left.G\left(\tau \omega_{p}\right)=(3 / 8) \tau \omega_{p}\right]$.

Some theoretical values have been plotted in Fig. 16(a), showing the good agreement with numerical results obtained by the Hilbert-Vlasov code. As before, in order to evaluate the transferred action to accelerated particles and to check the accuracy of the numerical simulation, we have represented in Fig. 16(b) the mean number of pump photons, $N_{0}$, of plasmons $N_{e}$, the wave-particle plasmons $N_{\mathrm{WP}}$, and their mutual sums $N_{2}=N_{0}+N_{e}+N_{\mathrm{WP}}$, versus time. Excellent agreement with the expected behavior $N_{2} / n_{0} c$ $=F_{0} G\left(t \omega_{p}\right) \hbar \omega_{p} / n_{0} m c^{3}$ is observed.

\section{NUMERICAL VLASOV SIMULATION OF CASCADE PROCESSES}

In this section we discuss cascade processes, a more complicated situation in which, if a sufficiently intense Stokes (or idler here) wave is generated, it can then act as a secondary pump wave and generates a higher-order Stokes shifted wave at frequency $\omega_{2 s}=\omega_{s}-\omega_{e}$ and so on. A whole hierarchy of higher-order Stokes/anti-Stokes sidebands can be generated in this fashion (however, the anti-Stokes wave is in this case attenuated rather than amplified). We show here the possibility of handling such a problem using the Hilbert-Vlasov model and make a comparison with the numerical results obtained with the full electromagnetic version of the Vlasov code.

A simulation was performed to illustrate an example of down-cascading of the idler wave to a second Stokes component in the case of a beatwave experiment. Because in this case we want to be able to compare the results with those given by the Maxwell-Vlasov code, we choose a modest pump wave frequency $\omega_{0}=3.66 \omega_{p}$ (leading to a one-step cascade process) in order to keep the computational burden from being too prohibitive. The simulation parameters are summarized in Table V.

\section{A. Basic equations}

As before, we consider two copropagating laser beams with frequencies and wave numbers $\left(\omega_{0}, k_{0}\right)$ (for the pump wave) and $\left(\omega_{s}, k_{s}\right)$ (for the idler wave) resonantly drive a plasma wave with frequency $\omega_{e}=\omega_{0}-\omega_{s}$ and wave number $k_{e}=k_{0}-k_{s}$. Now, however, the cascade of the idler wave

TABLE V. Numerical parameters used in the Vlasov simulations to illustrate an example of down-cascading of

\begin{tabular}{|c|c|c|c|c|c|}
\hline & $\begin{array}{l}\text { Pump wave } \\
\text { (0) }\end{array}$ & $\begin{array}{l}\text { Idler wave } \\
\text { (Stokes) }(s)\end{array}$ & $\begin{array}{l}\text { Plasma wave } \\
\text { (Stokes) }(e)\end{array}$ & $\begin{array}{c}\text { Second } \\
\text { Stokes }(2 s)\end{array}$ & $\begin{array}{l}\text { Plasma wave } \\
\text { (Cascade) }\left(e^{\prime}\right)\end{array}$ \\
\hline$\omega / \omega_{p}$ & 3.661 & 2.608 & 1.0531 & 1.543 & 1.064 \\
\hline$k c / \omega_{p}$ & 3.522 & 2.409 & 1.113 & 1.175 & 1.225 \\
\hline Velocity & $\begin{array}{c}0.077 \\
\text { (quiver) }\end{array}$ & $\begin{array}{l}0.108 \\
\text { (quiver) }\end{array}$ & $\begin{array}{l}0.946 \\
\text { (phase) }\end{array}$ & & $\begin{array}{l}0.868 \\
\text { (phase) }\end{array}$ \\
\hline Momentum & $\begin{array}{l}0.077 \\
\text { (quiver) }\end{array}$ & $\begin{array}{l}0.109 \\
\text { (quiver) }\end{array}$ & $\begin{array}{l}2.933 \\
\text { (phase) }\end{array}$ & & $\begin{array}{l}1.752 \\
\text { (phase) }\end{array}$ \\
\hline$\lambda \omega_{p} / c$ & 1.78 & 2.61 & 5.65 & 5.34 & 5.13 \\
\hline
\end{tabular}
the idler wave to a second Stokes wave in the case of a beatwave experiment. 
generates a scattered wave $\left(\omega_{2 s}, k_{2 s}\right)$ (which is here termed the second Stokes wave), and also a second plasma wave $\left(\omega_{e}^{\prime}, k_{e}^{\prime}\right)$, which is different from the first one, because of the difference in matching conditions $\omega_{s}=\omega_{2 s}+\omega_{e}^{\prime}$ and $k_{s}$ $=k_{2 s}+k_{e}^{\prime}$. A proper treatment that accounts for both idler and second Stokes scattering must include all three electromagnetic waves (that means pump, idler, and second Stokes waves) in the expression of the total vector potential:

$$
\begin{aligned}
A_{y}(x, t)= & \frac{1}{2} A_{0}(x, t) e^{i\left(k_{0} x-\omega_{0} t\right)}+\frac{1}{2} A_{s}(x, t) e^{i\left(k_{s} x-\omega_{s} t\right)} \\
& +\frac{1}{2} A_{2 s}(x, t) e^{i\left(k_{2 s} x-\omega_{2 s} t\right)}+\text { c.c. }
\end{aligned}
$$

For convenience we assume both plasma waves to be identical and include the difference explicitly in the small mismatch $\Delta k_{s}$ in the wave matching condition $k_{s}+\Delta k_{s}=k_{2 s}+k_{e}$, with $\Delta k_{s}=0.120 \omega_{p} / c$. This yields the following equations:

$$
\begin{aligned}
& \left(\frac{\partial}{\partial t}+\nu_{g 0} \frac{\partial}{\partial x}\right) A_{0}=-\frac{i \omega_{p}^{2}}{4 \omega_{0}} \rho_{e} A_{s}, \\
& \left(\frac{\partial}{\partial t}+\nu_{g S} \frac{\partial}{\partial x}\right) A_{s}=-\frac{i \omega_{p}^{2}}{4 \omega_{s}}\left(A_{0} \rho_{e}^{*}+A_{2 s} \rho_{e} e^{i \Delta k_{s} x}\right), \\
& \left(\frac{\partial}{\partial t}+\nu_{g 2 S} \frac{\partial}{\partial x}\right) A_{2 s}=-\frac{i \omega_{p}^{2}}{4 \omega_{2 s}} A_{s} \rho_{e}^{*} e^{-i \Delta k_{s} x} .
\end{aligned}
$$

Given complex electromagnetic wave envelopes by retaining only resonant and near-resonant terms, the calculation of the real ponderomotive force required by the Vlasov electrostatic code is then given by

$F(x, t)=\frac{e^{2}}{2 m} \frac{\partial\left(A_{y}^{2}\right)}{\partial x}=\frac{i k_{e} e^{2}}{4 m}\left(A_{0} A_{s}^{*}+A_{s} A_{2 s}^{*} e^{-i \Delta k_{s} x}\right)+$ c.c.

We now present the results of the numerical simulations.

\section{B. Numerical simulations of cascade processes}

Numerical simulations are performed for a plasma length of $L \omega_{p} / c \approx 456.5$ (256 pump wavelengths or about 12.6 plasma wavelengths). The wave number spectra of the transverse and of the longitudinal fields using the full electromagnetic version of the Vlasov code are illustrated in Fig. 17 at two instants. At both times, the idler and pump peaks are clearly resolved in the electromagnetic spectrum, and the peaks are in good agreement with the predictions obtained from the phase matching conditions and linear dispersion relations (see Table V). For $t \omega_{p}=200$, as expected, the second Stokes wave can be seen in the electromagnetic spectrum and the corresponding longitudinal electric field spectrum has distinct peaks at $k_{2 s} c / \omega_{p}=1.175$ and $k_{e}^{\prime} c / \omega_{p}=1.225$, respectively, with the latter wave from the cascade process now dominating the electrostatic results. [The electromagnetic spectra also exhibits an anti-Stokes component close to the theoretical value $k_{\mathrm{aS}} c / \omega_{p}=4.604$ (with a corresponding frequency $\omega_{\mathrm{aS}}=4.712 \omega_{p}$ ), but remains at a very low level. This component can be neglected in a
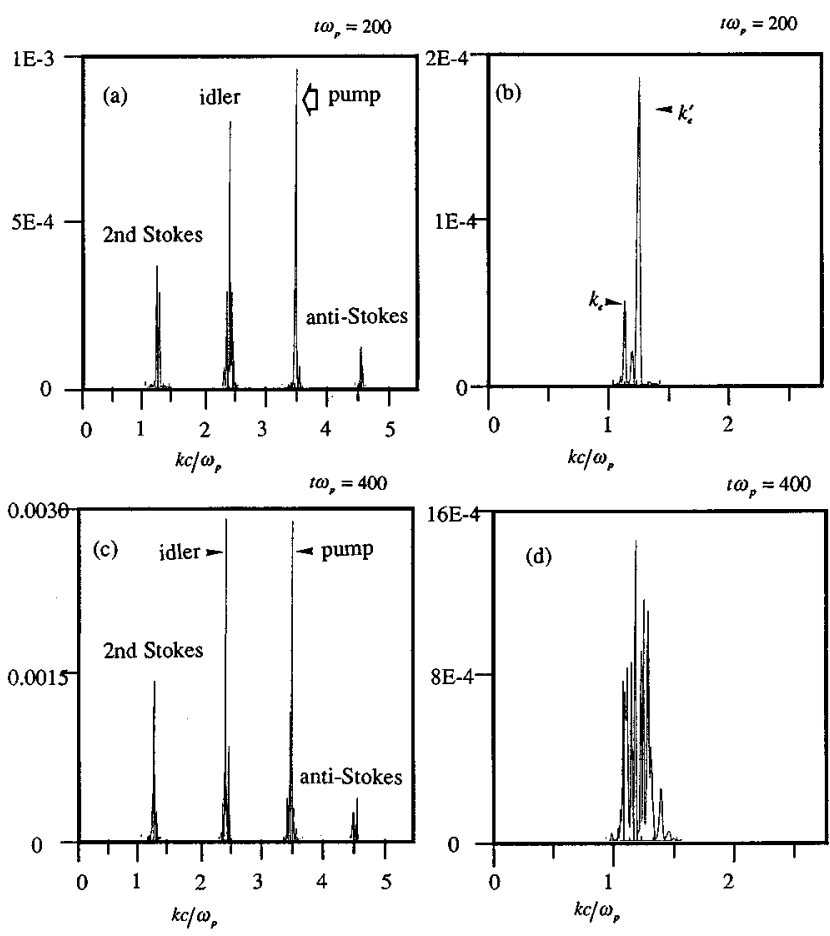

FIG. 17. One step-cascade wave number spectra from electromagnetic (a) and (c) and electrostatic [(b) and (d)] from the full electromagnetic version of the Vlasov code. Two different times, $t \omega_{p}=200$ and $t \omega_{p}=400$, are considered. The cascade of the idler wave generates a scattered wave (a second Stokes wave) of wave number $k_{2 s} c / \omega_{p}=1.175$ and also a second plasma wave of wave number $k_{e}^{\prime} c / \omega_{p}=1.225$. Note also the presence of an antiStokes component at the lower level and the plasma wave vector spectral broadening at the later time.

first step in the mode coupling analysis used in the HilbertVlasov, since this mode is not unstable.] Later, at time $t \omega_{p}=400$, the side peaks continue to grow and the $k$ spectrum of the plasma wave broadens significantly because of the increasing complexity in the plasma wave spatial structure. (The spectrum width remains, however, narrow enough to be treated accurately by a spatial Hilbert transform to compute the complex envelope of the electric field required in the mode-coupling mode.)

Figure 18 shows a portion of the phase space plots at a slightly later time $t \omega_{p}=500$ obtained by using the MaxwellVlasov code. The corresponding results given by the hybrid version are plotted in Fig. 19. The grey shade indicates the magnitude of the particle distribution function $f\left(x, p_{x}\right)$. As usual in this work, the solid curves mark the separatrix between the trapped and untrapped particle orbits for the local amplitude of the plasma wave. In spite of the fact that we have neglected the anti-Stokes contribution, the general features are well reproduced between both kinetic Vlasov codes. The spirals inside the separatrices implicitly reflect the history of the particles trapped as the wave built up. Note that these waves are not ultrarelativistic, so that the phase space structure is rather different from earlier structure met in Sec. $\mathrm{V}$. There are, however, some little modifications in the central region of the plasma, due to a large number of particle trapping/detrapping cycles, given more complexity in phase space but little change in momentum. In conclusion, coupling-mode equations can clearly be adapted to more 

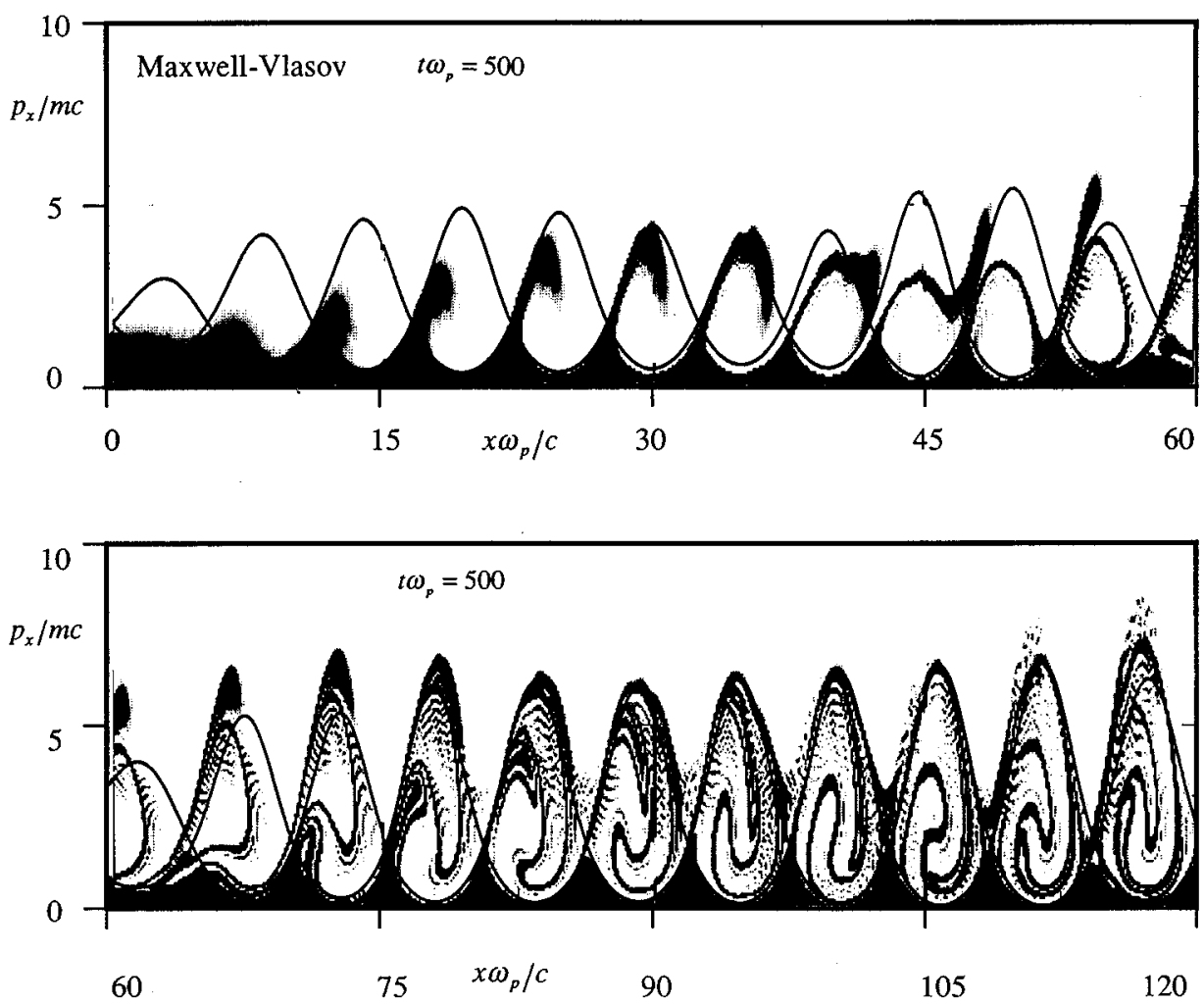

FIG. 18. For the full Vlasov code and the cascade simulation of Fig. 17, a region of the phase space at time $t \omega_{p}=500$.

complex and realistic situations, including more electromagnetic sidebands, but at high pump frequencies one must assess just how many electromagnetic modes will be included.

\section{CONCLUSION AND DISCUSSION}

Recent Eulerian Vlasov simulations of plasma have revealed a rich variety of phenomena associated with the fast particle dynamics induced by a beatwave experiment. However, these phenomena become very burdensome to treat numerically when electromagnetic frequencies are large compared with the plasma frequency. We have demonstrated an interesting possibility to extend such detailed modeling to frequency ratios greater than the current practical maximum of 10 or so for Vlasov or PIC (particle-in-cell) codes, by using mode equations for the electromagnetic waves rather than the full electromagnetic Vlasov codes. This is true, not only for studies related to beatwave acceleration, but also for stimulated Raman scattering or current drive in a magnetized plasma, or stimulated Brillouin scattering. The resulting hybrid version (which we called the Hilbert-Vlasov code) thus provides a saving of order $\left(\omega_{\text {pump }} / \omega_{\text {plasma }}\right)^{2}$ in computer time for forward processes, as compared with a direct attack by the full electromagnetic Maxwell-Vlasov method with the highest space-time resolution required.

To check the accuracy of the models, spatially integrated Manley-Rowe relations have been derived in a finite causal system in order to estimate the action transferred to fast particles. By studying the complex behavior of the coupled mode equations, we have thus been able to estimate the effects of the nonlinear absorption due to particle trapping with a view to developing better ways of estimating these losses and separating them from the coupled mode effects.

The electron dynamics in the very relativistic plasma waves generated by high-frequency beatwave drive are significantly different from what is seen at modest frequency ratios with lower phase velocities. In particular, there appears to be a significant bifurcation in particle trapping, with a significant fraction of electrons being relegated to momentum oscillations following just below the lower separatrix, without being trapped and accelerated, as in the case for electrons with favorable phasing. This result is somewhat unexpected, since it occurs even though the electron beam initial momentum is well above the lower separatrix trapping boundary if that could be instantaneously imposed. In future work, we hope also to examine how the particles exit the plasma wave resonant region and the final accelerated energy distribution.

The lower limit for the electron beam density in this simulation is set by the code precision, because electrons of only one kind are available at present. Using a separate electron species for the beam electrons would avoid this problem and allow simulation of lower beam densities closer to experimental estimates.

In this work, high-frequency backscatter was in effect forbidden, since no electromagnetic modes were provided for it. To include backscatter, one must not only introduce the 


\section{Phase-space representation}
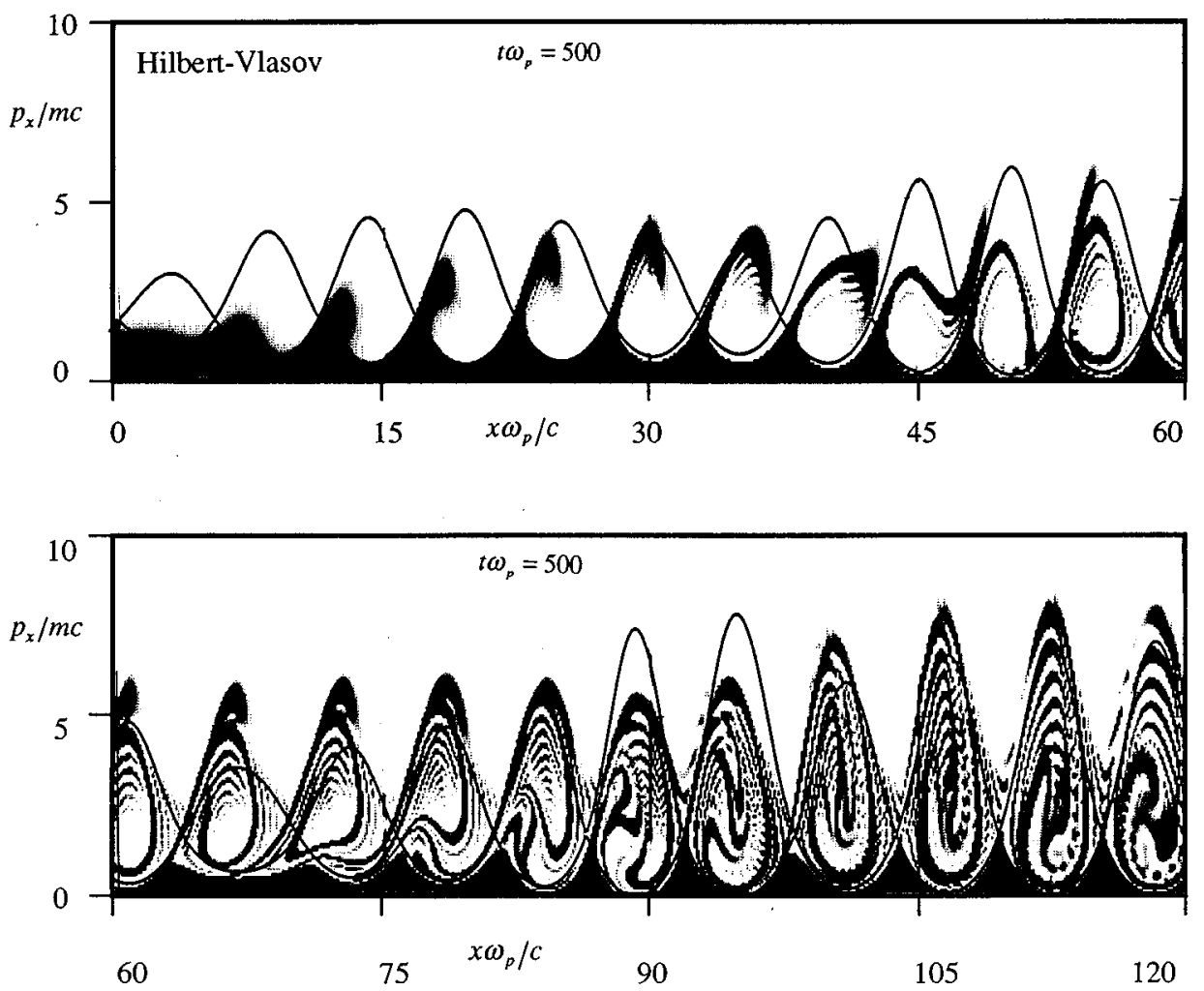

FIG. 19. The equivalent result to Fig. 18, but obtained now by the Hilbert-Vlasov model, in which Maxwell's equations have been replaced by the three-wave coupling model. While the particle acceleration features are, in a overall view, well reproduced by the model. There are, however, some distinct differences in the central region of plasma due to the number of complex particle trapping/detrapping cycles during the earlier evolution of the waves.

appropriate backscatter electromagnetic mode(s) (which is relatively inexpensive), but also provide sufficiently high spatial resolution for the plasma wave corresponding at least to the sum of the forward and backward wave numbers. In the case at hand, the computational saving over the full simulation would be more economical by only a factor $\left(\omega_{0} / \omega_{p}\right)$, rather than $\left(\omega_{0} / \omega_{p}\right)^{2}$ for the cases studied here. In spite of this increased computation burden, this is what might well have to be done to address some phenomenon seen in laser scattering results at UCLA (as yet unpublished) involving coupling between the driven beatwaves and plasma waves with backscatter wave numbers.

Furthermore, two-dimensional effects such as Raman sidescattering, filamentation, hydrodynamic expansion, and ponderomotive blowout are not considered in the present study, whereas they are, in fact, very important in the experiment. The Raman backscattering in an unmagnetized plasma may evolve into the sidescattering as the plasma begins to heat up and the damping for the backscattering increases. Also in the experiments, the plasma wave has a spatial profile in the direction of propagation of the laser beams due to the Gaussian optics. Thus, the rather good agreement between the energy gain seen in the code and in the experiment must be confirmed by a two-dimensional (2-D) Vlasov simulations. Although a 2-D Vlasov code version imposes a prohibitive computer loading and also computer burden, the Eulerian Vlasov method is probably much easier to realize in massively parallel computers than a PIC code (because one does not have to cope with the allocation of particles between processors, since the Eulerian elements stay in place). Furthermore, the efficiency lost of the Vlasov code for higher dimensions might be compensated for by the use of massively parallel computers. Both approaches (PIC and Vlasov code) require a more detailed comparison on the interplay between physics aspects of the model on the one hand and performance and optimization issues on the other. An effort to accomplish this is well in hand and the results will be reported in due course.

\section{ACKNOWLEDGMENTS}

The authors are indebted to the IDRIS center ("Institut du Développement et des Ressources en Informatique Scientifique-Paris, France) for computer time allocation on the Cray-C98 and C94 computers. A. Ghizzo, P. Bertrand, and J. Lebas would like to acknowledge the hospitality of the Centre Canadien de Fusion Magnétique of Varennes and the Institut National de la Recherche Scientifique (INRS-Energie et Matériaux) of Varennes (Canada). They are grateful to M. R. Feix, E. Fijalkow, and S. J. Karttunen, R. R. E. Salomaa, and T. J. H. Pättikangas for very helpful discussions.

T. W. Johnston acknowledges support from the Ministère de l'Education du Québec and of the Canadian National Science and Engineering Research Council. 
${ }^{1}$ C. E. Clayton, K. A. Mars, A. Dyson, M. Everett, A. Lal, W. P. Leemans, R. Williams, C. Joshi, Phys. Rev. Lett. 70, 37 (1993), see also C. E. Clayton, M. J. Everett, A. Lal, D. Gordon, K. A. Marsh, and C. Joshi, Phys. Plasmas 1, 1753 (1994); also see J. S. Wurtele, Phys. Today 47, 33 (1994).

${ }^{2}$ F. Amiranoff, M. Laberge, J. R. Marquès, F. Moulin, E. Fabre, B. Cros, G. Matthieussent, P. Benkheiri, F. Jacquet, J. Meyer, P. Miné, and C. Stenz, Phys. Rev. Lett. 68, 3713 (1992).

${ }^{3}$ A. Ghizzo, P. Bertrand, M. Shoucri, T. W. Johnston, E. Fijalkow, and M. R. Feix, J. Comput. Phys. 90, 431 (1990).

${ }^{4}$ A. Ghizzo, T. Reveillé, P. Bertrand, T. W. Johnston, J. Lebas, and M. Shoucri, J. Comput. Phys. 118, 356 (1995).

${ }^{5}$ T. W. Johnston, P. Bertrand, A. Ghizzo, M. Shoucri, E. Fijalkow, and M. R. Feix, Phys. Fluids B 4, 2523 (1992).
${ }^{6}$ A. Ghizzo, P. Bertrand, J. Lebas, T. W. Johnston, and M. Shoucri, “A hybrid Eulerian Vlasov code. II. Numerical simulation of chirped beatwave experiment," to be submitted for publication in Phys. Plasmas (companion paper referred to as II).

${ }^{7}$ P. Bertrand, A. Ghizzo, T. W. Johnston, M. Shoucri, E. Fijalkow, and M. R. Feix, Phys. Fluids B 4, 2665 (1992).

${ }^{8}$ P. Bertrand, A. Ghizzo, S. J. Karttunen, T. J. H. Pättikangas, R. R. E. Salomaa, and M. Shoucri, Phys. Fluids B 4, 3590 (1992).

${ }^{9}$ T. Reveillé, P. Bertrand, A. Ghizzo, J. Lebas, T. W. Johnston, and M. Shoucri, Phys. Fluids B 4, 2665 (1992).

${ }^{10}$ M. N. Rosenbluth and C. S. Liu, Phys. Rev. Lett. 29, 701 (1972).

${ }^{11}$ P. Mora, Phys. Fluids B 4, 1630 (1992).

${ }^{12}$ D. L. Bruhwiler and J. R. Cary, Phys. Rev. Lett. 68, 255 (1992). 